\title{
How Specific Is Source Memory for Faces of Cheaters? Evidence for Categorical Emotional Tagging
}

\author{
Raoul Bell and Axel Buchner \\ Heinrich-Heine-Universität Düsseldorf \\ Trang Giang \\ Heinrich-Heine-Universität Düsseldorf
}

\author{
Edgar Erdfelder \\ Universität Mannheim
}

Cécile Schain

Westfälische Wilhelms-Universität Münster

\author{
Nina Riether \\ Universität Bielefeld
}

\begin{abstract}
Two experiments designed to examine the specificity of emotional source memory are reported. In the encoding phase, participants saw faces along with emotional context information, that is, descriptions of cheating, trustworthy, or irrelevant behavior. In the test phase, participants were required to complete a source classification test and a cued recall test. In both experiments, the source memory advantage for faces characterized by negative context information (cheating) was replicated. Extending previous research, a multinomial source-monitoring model was applied to distinguish between specific source memory for individual behavior descriptions and partial source memory in the sense of only a rough classification of the behavior as belonging to a particular emotional category-cheating, trustworthy, or neither of these. The results indicate that the source memory advantage for the emotional context information is not always accompanied by enhanced recollection of the specific details of the learning episode and might rather reflect unspecific memory for categorical emotional information.
\end{abstract}

Keywords: memory specificity, emotional source memory enhancement, context memory, emotion and memory, multinomial model

The present study examines the qualitative nature of emotional source memory. The term source memory refers to the ability to remember the context in which information was learned and is used to describe a large range of phenomena, such as memory for the association of a stimulus with its location, time of occurrence, perceptual attributes, and other stimuli present in the learning environment. The presence or absence of memory for episodic context seems to discriminate between recollective experiences and familiarity-based memory judgments (Meiser \& Bröder, 2002; Meiser \& Sattler, 2007; Meiser, Sattler, \& Weisser, 2008; Perfect,

This article was published Online First October 24, 2011.

Raoul Bell, Axel Buchner, and Trang Giang, Department of Experimental Psychology, Heinrich-Heine-Universität Düsseldorf, Düsseldorf, Germany; Edgar Erdfelder, Department of Psychology, Universität Mannheim, Mannheim, Germany; Cécile Schain, Department of Psychology, Westfälische Wilhelms-Universität Münster, Münster, Germany; Nina Riether, Research Institute for Cognition and Robotics, Universität Bielefeld, Bielefeld, Germany.

The research in this article was supported by a grant from the Deutsche Forschungsgemeinschaft (BU 945/7-2). We thank S. Sieberts for her help with data acquisition, and we thank Iris Mund for her help with propositional scoring.

Correspondence concerning this article should be addressed to Raoul Bell, Department of Experimental Psychology, Heinrich-HeineUniversität, D-40225 Düsseldorf, Germany. E-mail: raoul.bell@hhu.de
Mayes, Downes, \& Van Eijk, 1996). "Remember" judgments in the remember-know paradigm are often associated with better source memory for perceptual attributes of the stimuli (Meiser \& Bröder, 2002; Meiser \& Sattler, 2007; Meiser et al., 2008; Perfect et al., 1996), and conditions that enhance recollection also lead to enhanced source memory (Conway \& Dewhurst, 1995). In addition to overall differences in source memory between "remember" and "know" items, Meiser and colleagues (Meiser \& Sattler, 2007; Meiser et al., 2008) found qualitative differences in source memory between these two types of items. For "remember" items, but not for "know" items, source memory for two different context attributes was correlated. This suggests that different types of source memory exist that are related to different types of recollective experiences.

Despite its strong link to recollection, source memory may also rely on more diffuse states of knowing (Hicks, Marsh, \& Ritschel, 2002; Meiser \& Sattler, 2007). Hicks et al. (2002) found that items for which the source was correctly remembered were equally likely to be assigned to the "remember" or the "know" category (see also Conway \& Dewhurst, 1995), which suggests that source memory is not always accompanied by a vivid recollective experience. Hicks et al. proposed that "know" responses may be associated with incomplete or partial source memory (see also Meiser \& Sattler, 2007). Models of partial source memory (Dodson, Holland, \& Shimamura, 1998; Klauer \& Wegener, 1998) take into account that source memory may sometimes reflect recollections 
of specific details of the encoding contexts and sometimes only rough classifications of contexts as belonging to particular categories. For instance, in the "Who said what?" paradigm (Klauer \& Ehrenberg, 2005; Klauer \& Wegener, 1998; Klauer, Wegener, \& Ehrenberg, 2002), participants may remember the particular person who made a verbal statement. However, even when this specific information is lost, participants may still remember whether the person who made the statement was male or female. One reason why it is interesting to assess partial source memory in the "Who said what?" paradigm is that it is revealing about how people categorize their social environment (Klauer \& Ehrenberg, 2005; Klauer \& Wegener, 1998; Klauer et al., 2002).

In contrast to the studies just mentioned, the present study addresses the specificity of source memory for emotional contexts. Source memory is often enhanced for emotional items. For instance, source memory for the font color of emotion words was found to be enhanced (D'Argembeau \& Van der Linden, 2004; Doerksen \& Shimamura, 2001; Kensinger \& Corkin, 2003). However, source memory for other contextual aspects was impaired by emotion (Anderson \& Shimamura, 2005; Cook, Hicks, \& Marsh, 2007; Mather et al., 2006). Based on these discrepant findings, it has been suggested that the effects of emotion on source memory may differ depending on the type of source memory being assessed. Attention is drawn away from nonemotional background information, leading to decreased memory for nonemotional context information, and directed to the emotional event, leading to better memory for the details of the emotional event itself (Kensinger, 2007; Mather, 2007). This may include memory for the emotional context in which a neutral stimulus is presented.

Often, the emotional source memory advantage is accompanied by enhanced recollection. Kensinger and Corkin (2003) found that the memory enhancement for negative stimuli was mainly caused by an increase in "remember" judgments in the remember-know paradigm. At the same time, memory for the negative stimuli contained more contextual details than did memory for neutral events. They concluded that negative emotion enhanced the detailedness and vividness of memory. However, enhanced source memory for emotional information might not always be accompanied by increased detailedness and vividness of memory, as the present study will show. Here, we examine the specificity of source memory for faces characterized by negative, neutral, and positive behavior descriptions. Several recent studies have found a source memory advantage for faces of cheaters (i.e., enhanced memory for the cheating context in which these faces were encountered) over other types of faces (Bell \& Buchner, 2010a; Buchner, Bell, Mehl, \& Musch, 2009). These findings were interpreted as being consistent with social contract theory (Cosmides \& Tooby, 1992), which postulates that social exchange is so important to human evolution that specific brain mechanisms have evolved that help one to avoid exploitation by cheaters. Enhanced memory for faces of cheaters has been attributed to a highly specialized cheater detection module closely tied to the faceprocessing system (Mealey, Daood, \& Krage, 1996). However, the findings that the source memory advantage generalizes to stimuli other than faces (Bell \& Buchner, 2009) and other types of threatening context information (Bell \& Buchner, 2010b) suggest that the source memory advantage for faces of cheaters is due to prioritized processing of emotional, and especially negative, material, consistent with prior studies showing a source memory advantage for negative information (D'Argembeau \& Van der Linden, 2004; Doerksen \& Shimamura, 2001; Kensinger \& Corkin, 2003).

The source memory advantage for negative information is often attributed to enhanced recollection of the negative material (Kensinger \& Corkin, 2003; Ochsner, 2000). However, as yet it has not been examined whether the source memory advantage for faces of cheaters is accompanied by a detailed and vivid memory of the source information. In previous studies, the source memory test required participants to indicate whether a particular face belonged to a negative (cheating), neutral, or positive (trustworthy) context. Thus, the source memory advantage for faces of cheaters may in principle have been due to (1) memory for unspecific negative valence of the encoding context, (2) memory for categorical emotional information associated with the face, or (3) improved recollection of the specific behavior descriptions. A recent study (Bell, Giang, \& Buchner, 2010) exploring the qualitative aspects of emotional source memory found that people are able to discriminate between different types of negative contexts (e.g., descriptions of cheating and of disgusting behavior). This finding is clearly inconsistent with the first of the three possible explanations of the cheater advantage in source memory. Obviously, people do not remember only that a cheater face was associated with negative valence but are also able to retrieve information that is specific for the particular type of emotional context information. However, it is still unclear how specific memory for the behavior descriptions really is. The source memory advantage may be due to better recollection of the specific details of the negative context information (Explanation 3). For instance, when seeing the face of a cheater in the memory test, participants might recall that the face belonged to the used-car dealer who sold restored crashed cars as supposedly accident-free. Based on the detailed recollection of the emotional context information, participants would then conclude that the face belonged to a cheater. Alternatively, it is possible that the source memory advantage is caused by categorical emotional tagging (Explanation 2). Given that cheating and disgusting behavior elicit distinct emotional reactions (e.g., moral contempt and disrelish), participants might be able to correctly classify a face as a "cheater" because they are able to remember an association between a face and their emotional reaction to that face at encoding without recollecting the specific details of the context information that led to this emotional categorization.

From a functional perspective (e.g., Nairne, 2010; Nairne \& Pandeirada, 2010), it is open whether it should suffice to remember not to trust a particular person (regardless of the specific form of cheating the person was associated with) or whether it may confer additional advantages to recall the specific cheating behavior. The bulk of research has shown that antisocial and nonnormative behavior does not generalize across situations (e.g., Zimbardo, 2004) and that the cross-situational stability of cheating is questionable (e.g., Leming, 1978). For instance, somebody who is cheating in specific types of situations (e.g., somebody who refuses to pay taxes) might be a reliable cooperation partner in others (e.g., as a friend). Specific source memory for faces of cheaters might confer advantages because it enables the individual to take efficient countermeasures tailored to specific types of cheating (e.g., to count change if a clerk has a history of shortchanging but to trust his advice otherwise) rather than to take overly general precautions to avoid exploitation. Refusing to cooperate with all 
types of cheaters indiscriminately may incur costs because it may unnecessarily limit the opportunities to cooperate with these individuals. Remembering the specific type of cheating behavior would enhance the predictability of the interactants' behaviors and may thus help to avoid mistakenly rejecting reliable interaction partners.

However, remembering only the categorical information that someone is a cheater without remembering specific details would have the huge advantage of requiring fewer memory resources than would specific source memory. Limitations in cognitive resources put constraints on the amount of information that can be processed in social situations. It is therefore adaptive to rely on categorical information to integrate new experiences into existing semantic structures (Fiske \& Neuberg, 1990; Macrae \& Bodenhausen, 2000). Category formation is a decisive tool to build up well-organized memory structures that in turn facilitate information processing. Social categories render the world predictable and allow both fast and appropriate responses to social events (Van Knippenberg \& Dijksterhuis, 2000). In contrast, retrieving and integrating information from several different interaction episodes when a decision is required may take too much time to be of use in most social interactions (Klein, Cosmides, Tooby, \& Chance, 2002) and may be more susceptible to forgetting than simply storing and retrieving that someone is a cheater. Categorical processing will not always lead to the best decisions, because it implies that not all information is used; however, it enables efficient social decision making. Therefore, it may be beneficial to store only a summary judgment in the form of a categorical emotional tag.

In the present study, it was necessary, first, to replicate the source memory advantage for faces of cheaters observed previously (Bell \& Buchner, 2009, 2010a; Buchner et al., 2009). In contrast to previous studies using the same paradigm, here participants were required to recall the behavior descriptions accompanying the faces at encoding to examine whether the source memory advantage for faces of cheaters is due to (a) unspecific memory for categorical emotional information or (b) memory of the specific details of the context information.

\section{Experiment 1}

\section{Method}

Participants. Participants were 62 persons (32 women), most of whom were students at Heinrich-Heine-University Düsseldorf. Their ages ranged from 19 to 48 years $(M=26)$. They were paid for participating.

Materials. The same set of 72 facial photographs of men (256-bit, 116-pixel $\times$ 164-pixel grayscales) was used as in Buchner et al.'s (2009) experiments. For each participant, 36 of these faces were randomly selected for presentation in the encoding phase.

Descriptions typed below the photographs conveyed the behavioral history of the person. Twelve descriptions were selected for presentation in each condition of the behavioral-history variable (cheating, irrelevant, and trustworthy). These descriptions were identical to those used in previous studies examining source memory for faces of cheaters (Bell \& Buchner, 2010a; Buchner et al., 2009). The descriptions included information about the depicted person's profession. Given that the socioeconomic status conveyed by the professions did not modulate face recognition or source memory in several studies (Buchner et al., 2009; Mehl \& Buchner, 2008), it seemed justified to use only low-status professions to keep the design simple. For instance, "K. S. is a used-car dealer. He regularly sells restored crash cars as supposedly accident-free and conceals serious defects from the customers" would convey a history of cheating. "O. N. is a scaffolder. Presently, he works at a building site in southern Germany where several tenements and office buildings are to be built" would convey behavior that is irrelevant to the cheating-trustworthiness dimension. "O. D. is a cheese monger. He strongly attends to sorting out old cheese immediately and allows all his customers to try all his products" would convey trustworthy behavior. Ignoring the information about the persons' professions, all (German) behavior descriptions were 18 words long. Faces and descriptions were combined randomly for each participant.

Information about the valence of the descriptions was obtained in an independent norming study $(N=36)$ reported previously (Buchner et al., 2009). In this norming study, valence was assessed on a scale ranging from -3 (negative) to +3 (positive). An item-based analysis showed that cheating was perceived as being more negative than irrelevant behavior, $t(22)=15.25, p<.01, \eta^{2}=.91$, and trustworthiness was more positive than irrelevant behavior, $t(22)=25.22, p<$ $.01, \eta^{2}=.97$. In terms of absolute valence (i.e., ignoring the minus sign in the valence ratings), cheating and trustworthiness were equally valent, $t(22)=1.12, p=.27, \eta^{2}=.05$.

Two raters with expertise in propositional scoring (Bell, Buchner, \& Mund, 2008; Mund, Bell, \& Buchner, in press) performed a propositional analysis on the behavioral descriptions to assess the amount of information provided by the texts. The descriptions were decomposed into propositions according to Turner and Greene's (1977) scheme, which is based on Kintsch and van Dijk's (1978) text comprehension model. Disagreements were minimal and resolved through discussion. The number of propositions did not differ between conditions: that is, between cheating and trustworthiness, $t(22)=-0.83, p=.42, \eta^{2}=.03$; between cheating and irrelevant behavior, $t(22)=-0.50, p=.62, \eta^{2}=.01$; and between trustworthiness and irrelevant behavior, $t(22)=0.26, p=$ $.80, \eta^{2}=.02$ (see Table 1$)$.

Procedure. All participants were tested individually. They were required to rate the likability of 36 facial photographs presented in random order during the encoding phase. Each trial started with the presentation of a headline ("How likable do you find this person?") and a face. The behavior description was shown below the face $2 \mathrm{~s}$ later. The likability rating scale, ranging from 1 (not likable at all) to 6 (extremely likable) was presented $4.5 \mathrm{~s}$ later. Participants rated the likability using the computer mouse. The photograph and the behavior description stayed on screen until the participant initiated the next trial by clicking a continue button.

In the subsequent test phase, participants saw a random sequence of 72 faces, half of which had been presented in the encoding phase and half of which were new. Each trial started with the headline "Is this face old or new?" and a face. Participants selected an old or a new checkbox to indicate whether they had seen the face during the encoding phase or not. Following an "old" judgment and a click on the continue button, checkboxes labeled cheating, trustworthy, and neither cheating nor trustworthy appeared. Participants judged the behavior used in the description 
Table 1

Properties of the Behavior Descriptions Used in the Present Experiments

\begin{tabular}{|c|c|c|c|c|c|c|}
\hline \multirow{2}{*}{$\begin{array}{l}\text { Experiment and } \\
\text { variable }\end{array}$} & \multicolumn{2}{|c|}{ History of cheating } & \multicolumn{2}{|c|}{$\begin{array}{l}\text { Irrelevant } \\
\text { information }\end{array}$} & \multicolumn{2}{|c|}{$\begin{array}{l}\text { History of } \\
\text { trustworthiness }\end{array}$} \\
\hline & $M$ & $S D$ & $M$ & $S D$ & $M$ & $S D$ \\
\hline \multicolumn{7}{|l|}{ Experiment 1} \\
\hline Valence & -2.5 & 0.5 & 0.3 & 0.6 & 2.3 & 0.7 \\
\hline No. of propositions & 8.6 & 1.5 & 8.9 & 1.7 & 9.1 & 1.4 \\
\hline No. of words & 18.0 & & 18.0 & & 18.0 & \\
\hline No. of sentences & 1.0 & & 1.0 & & 1.0 & \\
\hline \multicolumn{7}{|l|}{ Experiment 2} \\
\hline Valence & -2.1 & 0.4 & 0.0 & 0.2 & 2.0 & 0.4 \\
\hline Concreteness & 0.9 & 0.7 & 0.9 & 0.4 & 0.9 & 0.5 \\
\hline No. of propositions & 3.2 & 0.3 & 3.2 & 0.4 & 3.1 & 0.4 \\
\hline No. of words & 7.1 & 1.1 & 7.2 & 0.8 & 7.2 & 0.7 \\
\hline No. of sentences & 1.0 & & 1.0 & & 1.0 & \\
\hline
\end{tabular}

Note. Values represent sample means (means of the items). Valence ratings ranged from -3 (negative) to +3 (positive). Concreteness ratings ranged from -3 (very abstract) to +3 (very concrete).

accompanying the face in the encoding phase. After selecting one of these checkboxes and clicking the continue button, a picture of a microphone appeared as a signal for participants to recall the behavior description associated with the face with as many details as possible. The computers' built-in microphones recorded the participants' answers. Clicking the continue button initiated the next trial.

Design. The within-subject independent variable was behavioral history (cheating, irrelevant, trustworthy). The dependent measures were likability ratings, old-new recognition, source memory, and recall of the specific behavior descriptions. A multivariate approach was used for all general linear model withinsubject comparisons. In the present application, all multivariate test criteria correspond to the same (exact) $F$ statistic, which is reported. Partial eta squared values are reported as a measure of effect size. The level of significance was set to $p<.05$ for all analyses. Source memory was analyzed using a multinomial processing tree model (see the Global Source Memory section of Results). Given a sample size of $N=62$ and $\alpha=.05$, the power to detect a difference between the source memory parameters for faces of cheaters and irrelevant faces $\left(d_{\mathrm{C}}\right.$ and $d_{\mathrm{I}}$, respectively) with an effect size of $w=0.06$ - which is in the order of magnitude of the source memory effect observed by Buchner et al. (2009)—was reasonably large $(1-\beta=.98)$. The same applies to the general linear model within-subject comparison, where an effect of size $f=.20$ could be detected with a probability of $1-\beta=.95$, assuming an average population correlation between the levels of the behavioral history repeated-measures variable of $\rho=.55$ (estimated from pilot data). The power calculation was conducted using $\mathrm{G}^{*}$ Power (Faul, Erdfelder, Lang, \& Buchner, 2007).

\section{Results}

Encoding-phase likability ratings. A repeated-measures multivariate analysis of variance (MANOVA) revealed a significant main effect of behavioral history on encoding-phase likability ratings, $F(2,60)=155.15, p<.01, \eta_{\mathrm{p}}^{2}=.84$. Orthogonal contrasts showed that faces of cheaters were less likable than other types of faces, $F(1,61)=83.80, p<.01, \eta_{\mathrm{p}}^{2}=.82$, and irrelevant characters were less likable than trustworthy characters, $F(1$, $61)=118.97, p<.01, \eta_{\mathrm{p}}^{2}=.66$. Mean likability ratings were 2.15 $(S E=0.08)$ for cheaters, $3.71(S E=0.07)$ for irrelevant characters, and $4.49(S E=0.08)$ for trustworthy characters.

Old-new recognition. Next, we examined old-new recognition by analyzing the hit rate (given that there was only one set of faces in the test phase, there was only one false alarm rate for all types of faces, so sensitivity measures would be redundant). Replicating the results of most previous studies examining old-new recognition for faces of cheaters (Barclay \& Lalumière, 2006; Bell \& Buchner, 2009, 2010a, 2011; Buchner et al., 2009; Mehl \& Buchner, 2008), a MANOVA showed that behavioral history had no effect on old-new recognition, $F(2,60)=2.21, p=.12, \eta_{\mathrm{p}}^{2}=$ .07. The mean hit rate was .63 $(S E=.03)$ for faces of cheaters, .65 $(S E=.03)$ for faces of irrelevant characters, and $.60(S E=.03)$ for faces of trustworthy characters. The mean false alarm rate was .04 $(S E=.01)$.

Global source memory. First, we tested whether the global source memory advantage for faces of cheaters could be replicated in the present experiment. When comparing source memory for different types of contexts, it is important to use a source memory measure that does not confound source memory with guessing biases. It is known that many ad hoc measures of source memory such as the number of correct source classifications or the conditionalized source identification measure (CSIM; defined as the number of correct source classifications conditionalized on the number of hits for a particular type of stimulus; Bröder \& Meiser, 2007; Murnane \& Bayen, 1996) confound source memory with guessing biases (Bayen, Murnane, \& Erdfelder, 1996; Bröder \& Meiser, 2007; Murnane \& Bayen, 1996). To illustrate, suppose that a participant has no source memory at all and decides to respond "cheater" to each of the 72 stimuli. As a result, all cheater faces would be correctly classified. Hence, both the number of correct source classifications and the CSIM would indicate perfect source memory for cheaters, which obviously would be false because source memory for cheaters was in fact nonexistent. This occurs because these ad hoc source memory measures imply unrealistic assumptions such as the assumption that guessing does not occur. 
Therefore, it is preferable to use a measurement model that takes guessing into account.

As a first step, we report the so-called unbiased hit rate proposed by Wagner (1993), which was previously used to measure source memory while taking guessing tendencies into account (Suzuki \& Suga, 2010). The unbiased hit rate $\left(H_{\mathrm{u}}\right)$ may be defined as the joint probability of correctly classifying a stimulus given that it is presented and of correctly using a response category given that it is used. For instance, the unbiased hit rate for the cheater faces is given by the proportion of cheater faces correctly classified as "cheaters" to the total number of cheater faces presented, multiplied by the proportion of cheater faces correctly classified as "cheaters" to the total number of faces classified as "cheaters," that is, by

$$
\begin{aligned}
& \frac{f_{\text {CheatCheat }}}{\left(f_{\text {CheatCheat }}+f_{\text {Cheatlrrelavant }}+f_{\text {CheatTrust }}+f_{\text {Cheat New }}\right)} \\
& \quad \times \frac{f_{\text {CheatCheat }}}{\left.f_{\text {CheatCheat }}+f_{\text {IrrelavantCheat }}+f_{\text {TrustCheat }}+f_{\text {NewCheat }}\right)},
\end{aligned}
$$

where $f_{\mathrm{ij}}$ is the frequency of responses of type $j$ to items of type $i$. The measure corrects for response biases toward particular stimulus categories by taking into account both stimulus frequency and response frequency.

Table 2 summarizes mean unbiased hit rates as a function of behavioral history. A MANOVA showed a main effect of behavioral history, $F(2,60)=4.88, p=.01, \eta_{\mathrm{p}}^{2}=.14$. Post hoc contrasts showed that source classifications were more accurate for faces of cheaters than for irrelevant faces, $F(1,61)=9.92, p<$ $.01, \eta_{\mathrm{p}}^{2}=.14$. Participants did not differ significantly in classifying faces of trustworthy and irrelevant characters, $F(1,61)=1.64$, $p=.21, \eta_{\mathrm{p}}^{2}=.03$. This replicates the pattern of findings obtained in previous studies (Bell \& Buchner, 2010a; Buchner et al., 2009).

However, it may be preferable to measure source memory using a better validated approach. Ideally, the measurement model should have been empirically validated by studies showing that the measurement model's source memory measures remain unaffected by differing levels of old-new recognition and response biases. Therefore, the results of the source classification test were analyzed using the well-validated multinomial source monitoring model (Bayen et al., 1996) that was successfully applied in previous studies to examine source memory for faces of cheaters (Bell \& Buchner, 2009, 2010a, 2011; Buchner et al., 2009) and for many other types of materials (e.g., Bayen \& Kuhlmann, 2011; Bayen, Nakamura, Dupuis, \& Yang, 2000; Bell et al., 2008; D’Argembeau \& Van der Linden, 2004; Ehrenberg \& Klauer, 2005; Meiser et al., 2008; Riefer, Chien, \& Reimer, 2007; Spaniol \& Bayen, 2002;

Table 2

Mean Unbiased Hit Rates $\left(H_{U}\right)$ for the Source Classification

\begin{tabular}{|c|c|c|c|c|}
\hline \multirow[b]{2}{*}{ Variable } & \multicolumn{2}{|c|}{ Experiment 1} & \multicolumn{2}{|c|}{ Experiment 2} \\
\hline & $M$ & $S E$ & $M$ & $S E$ \\
\hline History of cheating & .20 & .02 & .18 & .02 \\
\hline Irrelevant information & .12 & .02 & .12 & .02 \\
\hline History of trustworthiness & .16 & .02 & .16 & .02 \\
\hline
\end{tabular}
Performance as a Function of Behavioral History
Vogt \& Bröder, 2007). Multinomial measurement models have many advantages and have become popular in many areas, including source-monitoring research (for a review, see Erdfelder et al., 2009). The model is shown in Figure 1. Validation experiments (Bayen et al., 1996) have shown that the model provides a measure of source memory that is uncontaminated by old-new recognition and guessing biases. Recently, Schütz and Bröder (2011) have shown empirically that the Bayen et al. (1996) model is at least as good as an alternative model based on signal detection theory (see also Klauer \& Kellen, 2010). The model applied in the present study contains 12 free parameters, each of which represents the probability of certain cognitive processes. To illustrate, consider the first model tree in Figure 1, which represents processes prompted by test faces that correspond to cheaters. Parameter $D_{\mathrm{C}}$ represents the probability of recognizing a cheater face $(\mathrm{C})$ as old. Parameter $d_{\mathrm{C}}$ represents the conditional probability of also remembering that the face belonged to a cheater. If the source of a recognized face is not known (with probability $1-d_{\mathrm{C}}$ ), it may be guessed that the face belonged to a cheater with probability $a_{\mathrm{CT}} \times$ $a_{\mathrm{C}}$, to a trustworthy person with probability $a_{\mathrm{CT}}\left(1-a_{\mathrm{C}}\right)$, or to an irrelevant person with probability $\left(1-a_{\mathrm{CT}}\right)$. If a cheater face is not recognized as old (with probability $1-D_{\mathrm{C}}$ ), it may still be guessed, with probability $b$, that the face is old. For these faces, it may be guessed that the face belonged to a cheater with probability $g_{\mathrm{CT}} \times$ $g_{\mathrm{C}}$, to a trustworthy person with probability $g_{\mathrm{CT}}\left(1-g_{\mathrm{C}}\right)$, or to an irrelevant person with probability $1-g_{\mathrm{CT}}$. With probability $1-b$, the face is incorrectly judged to be new. Analogous statements hold for the model trees for trustworthy (T), irrelevant (I), and new (N) faces. Based on the model equations and the empirically observed sample responses to the different types of faces, the model parameters can be estimated (Erdfelder et al., 2009; Riefer $\&$ Batchelder, 1988). The multinomial analysis was conducted using multiTree (Moshagen, 2010).

We started our analysis with a base model that builds on the previously established fact that old-new recognition did not differ as a function of behavioral history. Hence, all parameters representing old-new recognition for the different types of previously encountered (old) faces were equated. To obtain an identifiable model, the parameter representing the probability of detecting new faces was equated with the probability of detecting old faces $\left(D_{\mathrm{C}}=D_{\mathrm{I}}=D_{\mathrm{T}}=D_{\mathrm{N}}\right)$. This is the standard assumption of two high-threshold models of signal detection that have been favorably evaluated in validation studies (Bayen et al., 1996; Klauer \& Wegener, 1998; Meiser \& Bröder, 2002; Snodgrass \& Corwin, 1988). The base model fit the data, $G^{2}(3)=5.94, p=.11$. The upper panel of Figure 2 shows the source memory parameters obtained. Setting the source memory parameter for faces of cheaters equal to the source memory parameter for faces of irrelevant characters $\left(d_{\mathrm{C}}=d_{\mathrm{I}}\right)$ was clearly incompatible with the data, $\Delta G^{2}(1)=16.22, p<.01$, implying a source memory advantage for faces of cheaters over faces of irrelevant characters. In contrast, source memory did not differ between faces of trustworthy characters and faces of irrelevant characters, $\Delta G^{2}(1)=1.78, p=.18$. This pattern replicates the results of previous studies (Bell \& Buchner, 2009, 2010a; Buchner et al., 2009).

Partial and specific source memory. The most interesting question was whether the source memory advantage for faces of cheaters was accompanied by enhanced memory for the specific cheating behaviors associated with these faces. A preliminary 


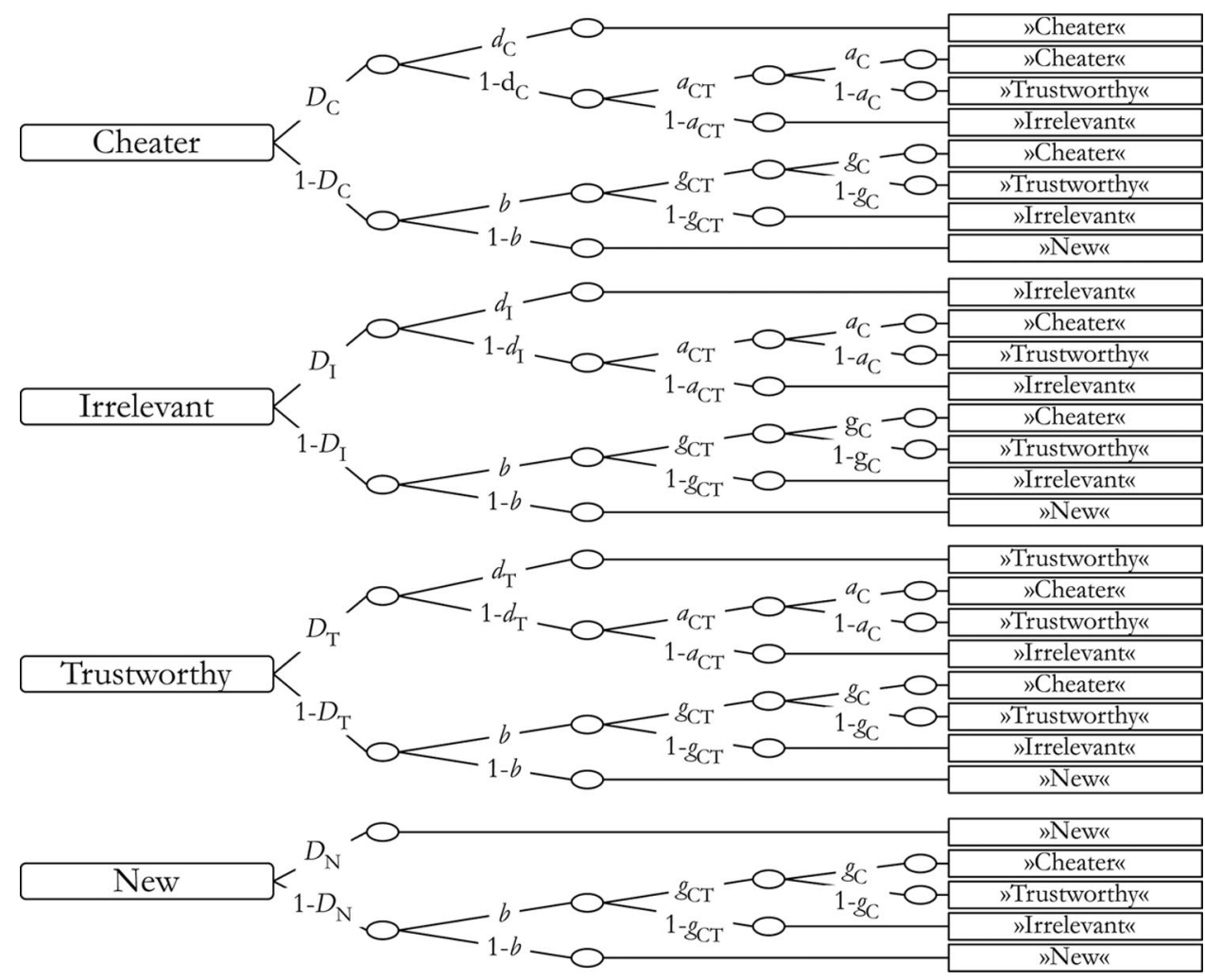

Figure 1. Bayen et al.'s (1996) source monitoring model. Rounded rectangles on the left side represent the types of faces presented (faces associated with cheating, irrelevant, and trustworthy behavior). Letters along the links represent the probabilities with which certain cognitive states occur. Rectangles on the right side represent participants' answers in the source classification task ("cheater," "irrelevant person," and "trustworthy person"). $D_{.}=$probability of correctly identifying a face as old or new; $\mathrm{C}=$ cheater; $\mathrm{I}=$ irrelevant; $\mathrm{T}=$ trustworthy; $\mathrm{N}=$ new; $d_{0}=$ source memory in the sense of remembering the type of behavior a face was associated with; $b=$ probability of guessing that a nonrecognized face is old; $a_{\mathrm{CT}}=$ probability of guessing that a recognized face was encountered in a socially relevant context (cheating or trustworthiness); $g_{\mathrm{CT}}=$ probability of guessing that a nonrecognized face was encountered in a socially relevant context; $a_{\mathrm{C}}=$ probability of guessing that a recognized face belonged to a cheater; $g_{\mathrm{C}}=$ probability of guessing that a nonrecognized face belonged to a cheater.

examination of the recall protocols showed that participants remembered almost no details of the behavioral descriptions. If participants remembered anything at all, they remembered the general theme (or the "gist") of the behavioral descriptions rather than their exact wording. For instance, rather than recalling "K. S. is a used-car dealer. He regularly sells restored crash cars as supposedly accident-free and conceals serious defects from the customers," participants typically recalled "He sells broken cars." The participants' answers were transcribed and compared with the behavior descriptions. It was judged whether the gist of the participants' answer was consistent with the gist of any of the behavior descriptions using a lenient scoring criterion. To evaluate the reliability of the scoring procedure, the recall protocols of 25 participants were judged by an independent rater. Interrater agreement as assessed by the kappa coefficient was $\kappa=.95$ (i.e., "almost perfect"; Landis \& Koch, 1977). Table 3 shows the proportion of faces for which the associated behavior description was remembered in the sense just described. Although there was a descriptive trend toward better memory for the gist of the cheating descriptions, memory for the specific behavior descriptions associated to the faces was low and did not differ among conditions, $F(2,60)=2.75, p=.07, \eta_{\mathrm{p}}^{2}=.08$. Thus, there was no significant memory advantage for the specific content of the cheater descriptions.

A further advantage of multinomial models is that they can be easily extended to assess different types of source memory varying in qualitative characteristics (Bröder \& Meiser, 2007; Dodson et al., 1998; Klauer \& Wegener, 1998; Meiser \& Bröder, 2002). To decompose participants' source memory into unspecific memory for the behavior category and specific memory for the behavior description, we analyzed the results of the memory test using a variant of the models of partial and specific source memory (Dodson et al., 1998; Klauer \& Wegener, 1998), which have been successfully applied in previous research (Klauer \& Ehrenberg, 2005; Klauer \& Wegener, 1998; Klauer et al., 2002). Figure 3 illustrates the first model tree representing the processes that are assumed to occur when a cheater face is presented at test. The model is identical to the source-monitoring model of Bayen et al. 
A

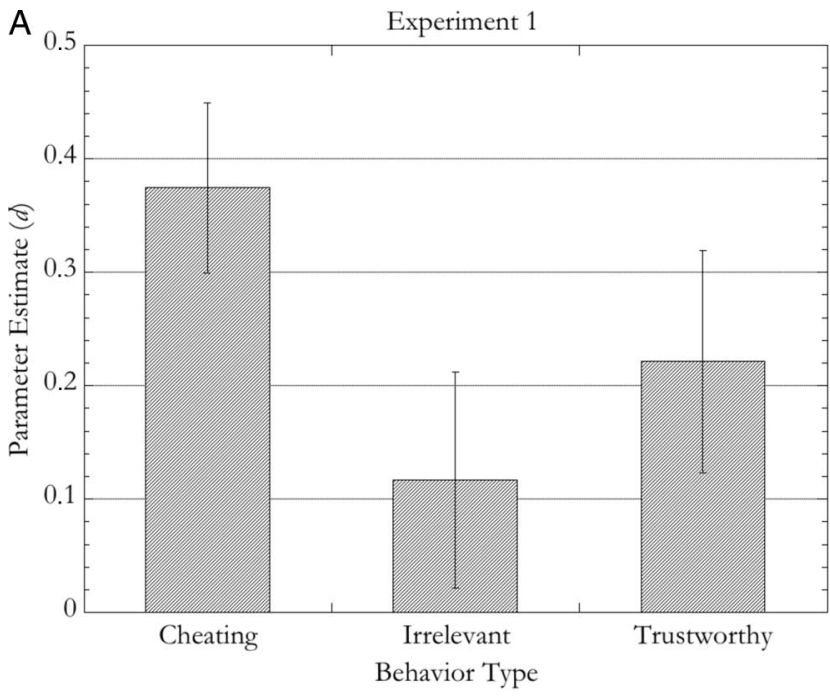

B

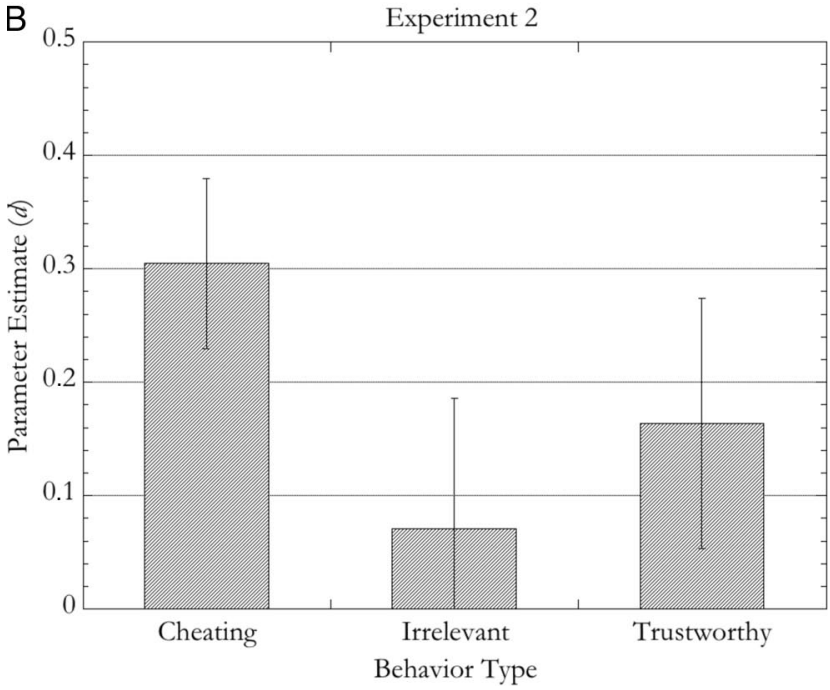

Figure 2. Parameter estimates for the source memory parameters for faces associated with cheating $\left(d_{\mathrm{C}}\right)$, for faces associated with irrelevant information $\left(d_{\mathrm{I}}\right)$, and for faces associated with trustworthiness $\left(d_{\mathrm{T}}\right)$ in Experiment 1 (Panel A) and 2 (Panel B). The parameters represent conditional probabilities of correct source identification given correct old-new recognition. Error bars represent the $95 \%$ confidence intervals.

(1996) described earlier (and illustrated in Figure 1), with two important exceptions. First, the model allows one to assess specific and partial source memory separately. It is assumed that people may have specific source memory for the behavior description (with probability $S_{\text {e, }}$, where the dot is to be replaced by $C, I$, or $T$ representing cheater, irrelevant, or trustworthy behaviors, respectively), which means that they are able to recall the gist of the specific behavior description accompanying the face at encoding. Alternatively, participants may have no memory for the specific behavior that the face was associated with (with probability $1-S$.) but may still be able to remember the behavior category (with probability $P$.). Second, the probability of guessing that the face belonged to a specific behavior description has to be incorporated into the model. For instance, if a face was correctly identified as a cheater, but the specific behavior accompanying the face at encoding was not remembered, participants may still reproduce a random cheating behavior (with probability $G_{\mathrm{C}}$ ), which may match the correct cheating description by chance (i.e., with probability $1 / 12)$.

To illustrate, the model tree for faces of cheaters shown in Figure 3 is described in more detail. Parameter $D_{\mathrm{C}}$ represents the probability of recognizing a cheater face as old. Parameter $S_{\mathrm{C}}$ represents the conditional probability of remembering the specific cheating behavior associated with the face. If the specific cheating behavior is not remembered (with probability $1-S_{\mathrm{C}}$ ), participants may still have partial source memory, in the sense that they may be able to retrieve the correct behavior category (a "cheater" tag) with probability $P_{\mathrm{C}}$. If participants have partial but no specific source memory for a face, they may nevertheless reproduce a random cheating behavior (from the set of all cheating behaviors encountered) with probability $G_{\mathrm{C}}$, which may match the correct description by chance (with probability $1 / 12$ ). When participants have neither specific nor partial source memory (with probability [1 $\left.S_{\mathrm{C}}\right] \times\left[1-P_{\mathrm{C}}\right]$ ), the correct behavior category (cheating) may still be guessed with probability $a_{\mathrm{CT}} \times a_{\mathrm{C}}$. With probability $G_{\mathrm{C}}$, a random cheating behavior is reproduced based on guessing, which will match the correct description by chance (with probability $1 / 12)$. With probability $a_{\mathrm{CT}} \times\left(1-a_{\mathrm{C}}\right)$, it may be guessed that the face was encountered in a trustworthy context. Then, a random description of trustworthy behavior is guessed with probability $G_{\mathrm{T}}$. With probability $1-a_{\mathrm{CT}}$, it may be guessed that the face was encountered in an irrelevant context. Then, a description of irrelevant behavior is guessed with probability $G_{\mathrm{I}}$. If the cheater face is not recognized as old (with probability $1-D_{\mathrm{C}}$ ), it may still be guessed, with probability $b$, that the face is old. For these faces, it may still be guessed that the face belonged to a cheater with probability $g_{\mathrm{CT}} \times g_{\mathrm{C}}$. With probability $G_{\mathrm{C}}$, a random cheating behavior is guessed, which will match the correct description with probability $1 / 12$. Alternatively, it may be guessed that the face belongs to a trustworthy person with probability $g_{\mathrm{CT}} \times\left(1-g_{\mathrm{C}}\right)$. Then, with probability $G_{\mathrm{T}}$, a random description of trustworthiness may be guessed. Alternatively, it may be guessed with probability $1-g_{\mathrm{CT}}$ that the face belongs to an irrelevant person. Then, a description of irrelevant behavior may be guessed with probability $G_{\mathrm{I}}$. With probability $1-b$, the face is incorrectly classified as new.

Table 3

Results of the Cued Recall Test for Recalled Behaviors and Propositions

\begin{tabular}{llllll}
\hline & \multicolumn{2}{c}{ Experiment 1 } & & \multicolumn{2}{c}{ Experiment 2 } \\
\cline { 2 - 3 } \multicolumn{1}{c}{ Variable } & $M$ & $S E$ & & $M$ & $S E$ \\
\hline Proportion of correctly & remembered & behavior & descriptions & \\
History of cheating & .09 & .01 & .08 & .01 \\
Irrelevant information & .06 & .01 & .05 & .01 \\
History of trustworthiness & .06 & .01 & .05 & .01 \\
\hline
\end{tabular}

Proportion of correctly recalled propositions

\begin{tabular}{lllll} 
History of cheating & .01 & $<.01$ & .04 & .01 \\
Irrelevant information & .01 & $<.01$ & .03 & .01 \\
History of trustworthiness & .01 & $<.01$ & .02 & .01 \\
\hline
\end{tabular}




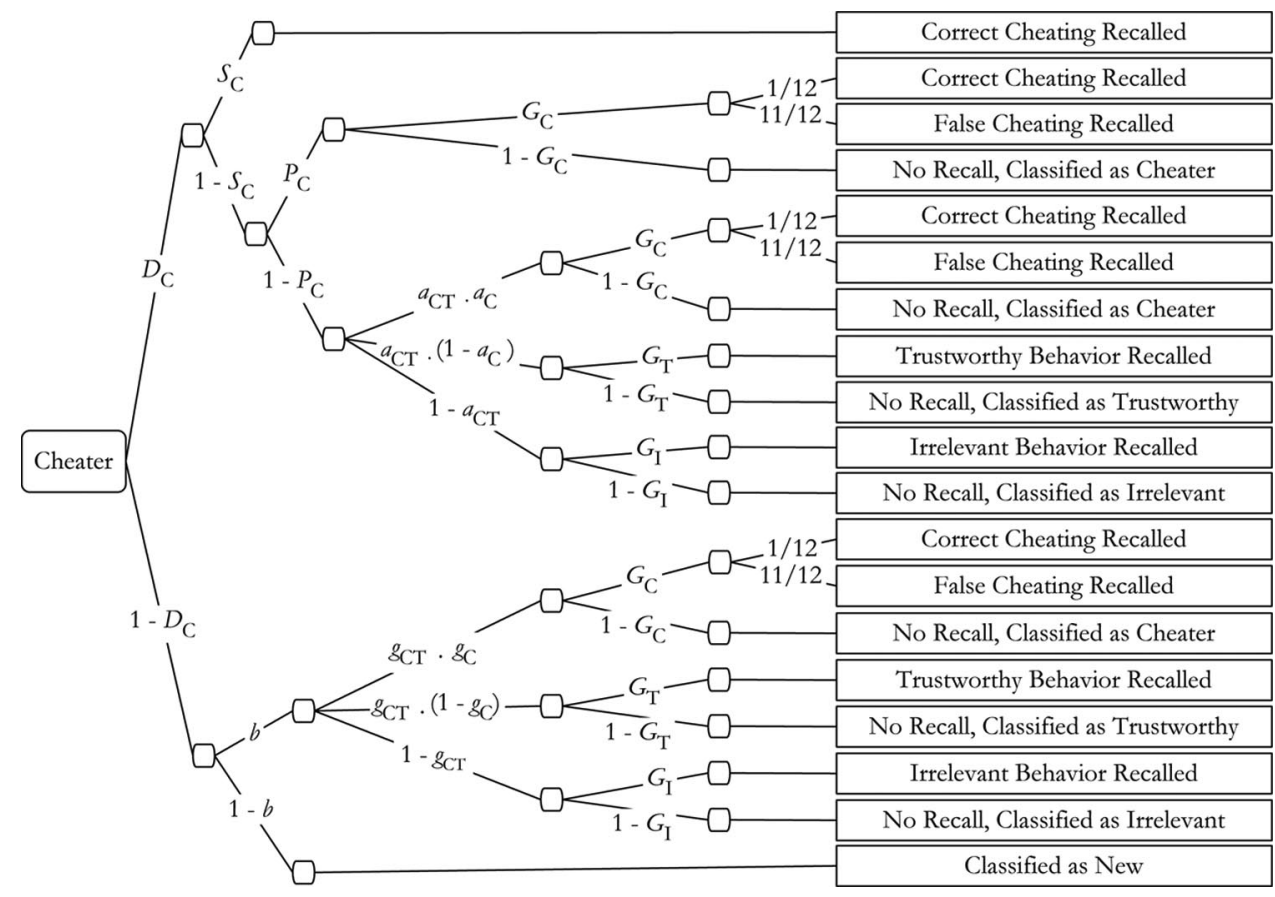

Figure 3. Illustration of the multinomial model used to separately assess partial and specific source memory. Only the model tree for the faces associated with cheating is shown. The rounded rectangle on the left side represents the type of faces presented (cheater faces). Letters along the links represent the probabilities with which certain cognitive states occur. The rectangles on the right side represent participants' answers in the source memory task. $D_{\mathrm{C}}=$ probability of correctly identifying a cheater face as old or new; $S_{\mathrm{C}}=$ specific source memory for the specific behavior description a cheater face was associated with; $P_{\mathrm{C}}=$ partial source memory for the behavior category a cheater face was associated with; $G_{\boldsymbol{0}}=$ probability of guessing a random behavior description when the specific behavior associated with the cheater face is not known; $b=$ probability of guessing that a nonrecognized face is old; $a_{\mathrm{CT}}=$ probability of guessing that a recognized face was encountered in a socially relevant context (cheating or trustworthiness); $g_{\mathrm{CT}}=$ probability of guessing that a nonrecognized face was encountered in a socially relevant context; $a_{\mathrm{C}}=$ probability of guessing that a recognized face belonged to a cheater; $g_{\mathrm{C}}=$ probability of guessing that a nonrecognized face belonged to a cheater.

Analogous considerations hold for the model trees for faces associated with descriptions of trustworthy and irrelevant behaviors and for new faces.

We again begin with a base model that implies that all parameters representing old-new discrimination are equal $\left(D_{\mathrm{C}}=D_{\mathrm{I}}=\right.$ $\left.D_{\mathrm{T}}=D_{\mathrm{N}}\right)$. The base model fit the data, $G^{2}(12)=18.01, p=.12$. The parameters for partial and specific source memory are displayed in Figure 4 (the full set of parameter estimates is given in the Appendix). At a descriptive level, the parameters of partial source memory for the behavior category showed the same pattern as the global source memory parameters in the previous analysis. Partial source memory was above zero in the cheater condition and in the trustworthy condition (i.e., the restriction that $P_{\mathrm{C}}=0$ and $P_{\mathrm{T}}=0$ were incompatible with the data, $\Delta G^{2}=36.73$, bootstrap $p<.01$, and $\Delta G^{2}=6.08$, bootstrap $p<.01$, respectively ${ }^{1}$ ). Consistent with the assumption that partial source memory reflects categorical emotional tagging, participants had no partial source memory for (nonemotional) irrelevant characters (i.e., the restriction that $P_{\mathrm{I}}=0$ was compatible with the data, $\Delta G^{2}=0.29$, bootstrap $p=.32$ ). Partial source memory was clearly better for faces of cheaters than for faces of irrelevant characters, $\Delta G^{2}(1)=$ $13.46, p<.01$. In contrast, partial source memory did not differ between trustworthy and irrelevant faces, $\Delta G^{2}(1)=1.81, p=.17$.
Next, we analyzed specific source memory for the behavior descriptions. Specific source memory was above zero in all conditions, which means that participants had specific source memory for all types of faces (i.e., the restrictions that $S_{\mathrm{C}}=0, S_{\mathrm{I}}=0$, and $S_{\mathrm{T}}=0$ were incompatible with the data, $\Delta G^{2}=163.80$, bootstrap $p<.01 ; \Delta G^{2}=171.88$, bootstrap $p<.01$; and $\Delta G^{2}=112.47$, bootstrap $p<.01$, respectively). Nevertheless, the absolute level of specific source memory was low in all conditions. Although specific source memory was somewhat better for cheater faces than for other faces at a descriptive level, it did not differ significantly between faces of cheaters and faces of irrelevant characters, $\Delta G^{2}(1)=2.94, p=.08$, and between faces of trustworthy characters and faces of irrelevant characters, $\Delta G^{2}(1)=0.01, p=.92$. This analysis suggests that the source memory advantage for faces of cheaters is mostly due to the retrieval of unspecific categorical information. Participants had enhanced source memory for faces of

\footnotetext{
${ }^{1}$ As the standard asymptotic chi-square goodness-of-fit test is not valid whenever the null hypothesis predicts parameter values at the boundary of the parameter space, we used the parametric bootstrap option of multiTree (Moshagen, 2010) to estimate $p$ values of $\Delta G^{2}$ statistics in these cases (see, e.g., Klauer \& Oberauer, 1995, for a similar treatment of this problem).
} 
A
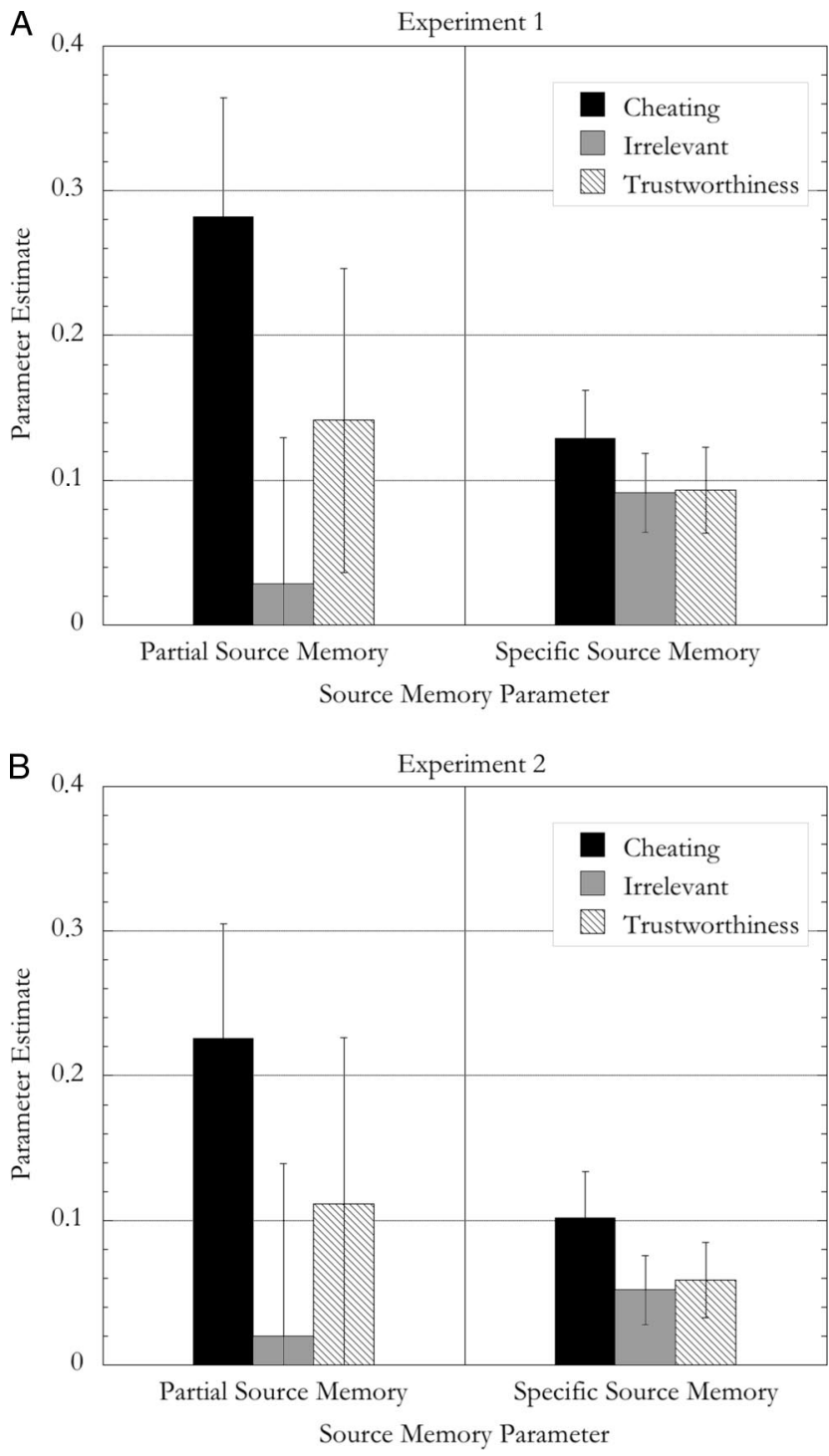

Figure 4. Left sides of panels: Parameter estimates for partial source memory for the behavior category accompanying a face at encoding (in the absence of memory for the specific type of behavior) for faces associated with cheating, faces associated with irrelevant information, and faces associated with trustworthiness in Experiment 1 (Panel A) and 2 (Panel B). Right sides of panels: Parameter estimates for specific source memory for the specific content of the behavior description accompanying a face at encoding for cheater faces, faces associated with irrelevant information, and faces associated with trustworthiness in Experiment 1 (Panel A) and 2 (Panel B). Error bars represent the 95\% confidence intervals.

cheaters because they succeeded in retrieving a "cheater" tag, but they failed to remember more specific information about the descriptions of cheating accompanying these faces at encoding.

Propositional recall. In the previous analysis, participants' answers were scored as matching the gist of the correct behavior description or not. This analysis was necessary to examine whether the source memory advantage for faces of cheaters was due to enhanced memory for the specific contents of the descriptions of cheating. However, this analysis ignores the amount of detail that is recalled from each behavior description. Based on evidence showing that negative arousal enhances the detail with which events are remembered (Kensinger, Garoff-Eaton, \& Schacter, 2006), one might be interested in whether individuals recalled more details about each cheater than about each irrelevant person. This hypothesis is not testable with the gist-based scoring strategy reported earlier. Therefore, an additional analysis was run in which we analyzed propositional recall to assess how much information from each behavior description was recalled. Propositions were scored following the procedure outlined by Turner and Greene (1977). Propositions comprising synonyms of to-be remembered words were scored as correct. To increase the reliability and validity of the scoring procedure, we used the Projekt Deutscher Wortschatz database (http://wortschatz.uni-leipzig.de/) to support the identification of synonyms. The database reflects knowledge about current-day word usage that is retrieved from a large collection of texts from diverse sources such as newspaper articles or web pages (Biemann, Bordag, Heyer, Quasthoff, \& Wolff, 2004). This procedure yielded good psychometric properties in a previous study (Mund et al., in press). Propositional recall as a function of behavioral history is displayed in Table 3. Participants recalled almost no propositions from the behavior descriptions. Propositional recall did not differ among conditions, $F(2,60)=0.23, p=$ $.79, \eta_{\mathrm{p}}^{2}<.01$. The source memory advantage for faces of cheaters was clearly not accompanied by a detailed recollection of the descriptions of cheating behavior. This analysis corroborates our conclusion that participants had almost no memory for the specific content of the behavior description to which a face was associated.

\section{Discussion}

The results of Experiment 1 are clear. First, the source memory advantage for faces of cheaters observed in previous studies (Bell \& Buchner, 2010a; Buchner et al., 2009) was replicated. Here we went one step further and analyzed whether this source memory advantage was (a) due to memory for the specific type of cheating conveyed by the behavior description or (b) due to partial source memory in the sense of a rough classification of the face as belonging to a cheater. The results suggest that the source memory advantage for faces of cheaters can largely be attributed to partial source memory for the fact that a person was encountered in a context of cheating in the absence of a detailed recollection of the specific cheating behavior accompanying the face at encoding. A memory advantage for faces of cheaters was obtained for partial source memory but not for specific source memory. Cued recall of the behavior descriptions lacked detail and did not vary significantly as a function of emotional content. We therefore concluded that the source memory advantage for faces of cheaters was largely due to the retrieval of unspecific categorical emotional information in the absence of a recollection of the specific details of the context information.

A methodological concern with studies such as the one presented here is always that differences among conditions might be attributed to confounds in the stimulus material, even though this hypothesis is unlikely in the present context for a number of reasons. First, memory for faces of cheaters and trustworthy and irrelevant persons has been examined using a carefully normed set of stimuli (Buchner et al., 2009; Mehl \& Buchner, 2008). Second, the source memory advantage for negative contexts was subse- 
quently replicated under a variety of conditions using a large variety of stimulus materials (Bell \& Buchner, 2009, 2010a, 2010b). Third, several alternative explanations have already been ruled out. For instance, the source memory advantage for faces of cheaters cannot be attributed to cheating being more exceptional or unusual than trustworthy or irrelevant behavior, because three experiments consistently showed that exceptionality of the behavior descriptions did not influence source memory for faces at all (Buchner et al., 2009). Fourth, the effect was found to depend on the participants' emotional sensitivity to injustice (Schmitt, Gollwitzer, Maes, \& Arbach, 2005). The source memory advantage was large in a group with high sensitivity to injustice but small and no longer statistically significant in a group with low sensitivity to injustice (Bell \& Buchner, 2010a). If the effect of behavioral history on source memory would only be caused by confounds in the stimulus material, there would be no reason why the source memory advantage should be modulated by sensitivity to injustice. Fifth, source memory for faces of perpetrators was found to be enhanced, whereas source memory for victims of cheating was not, even though the victim descriptions were virtually identical to the perpetrator descriptions except for the role of the stimulus person (Bell \& Buchner, 2011). These findings clearly show that the source memory advantage for faces of cheaters is not solely caused by confounds in the stimulus material. Nevertheless, it may be desirable to replicate the source memory effect using even more carefully controlled material. In Experiment 2, a new set of behavior descriptions was selected to control for a number of variables - such as length, valence, concreteness, and the number of propositions - that are known to influence memory.

\section{Experiment 2}

Experiment 2 served two purposes. The first purpose was to replicate conceptually the source memory advantage for faces of cheaters observed in Experiment 1 and elsewhere (Bell \& Buchner, 2010a; Buchner et al., 2009) using different stimulus materials. The second purpose of Experiment 2 was to examine whether the relative contribution of partial and specific source memory to the source memory advantage for faces of cheaters would be modulated by informational load. Emotional tagging may be a strategy that is preferentially used to reduce the constraints on memory when there is too much information available. When memory resources are overloaded, participants might resort to encoding the behaviorally relevant aspects of the behavior description in the form of an emotional tag. However, when the context information already corresponds to a composite summary of the descriptions used in Experiment 1, the need to reduce informational load may be lower and the memory for the specific details of the behavior descriptions may be higher. To test these hypotheses, we drastically reduced the length of the behavior descriptions from 18 words in Experiment 1 to seven words in Experiment 2. If the specificity of memory depends on the amount of information available (as assumed here), we would expect to find higher levels of memory for the specific details of the behavior descriptions in Experiment 2 compared with Experiment 1. Furthermore, we would expect to find a memory advantage for the specific details of the descriptions of cheating in comparison to other types of descriptions. However, if categorical emotional tagging is a more general phenomenon that can be observed whenever emotional contexts are remembered, we would expect to replicate the finding of Experiment 1 that the source memory advantage for faces of cheaters is due to unspecific memory for the emotional category without recollection of the specific details of the behavior descriptions.

\section{Method}

Participants. Participants were 47 persons (30 women), most of whom were students at Heinrich-Heine-University Düsseldorf. Their age ranged from 19 to 53 years $(M=26)$. They were paid for participating.

Materials, procedure, and design. Materials, procedure, and design were identical to those of Experiment 1 with the following exceptions. A new set of 12 descriptions was selected for each category of the behavioral history variable (cheating, irrelevant, trustworthy) based on the results of a norming study $(N=25$; see Table 1). The descriptions were much shorter than those used in Experiment 1. In German, all descriptions had a similar length and grammatical structure and conveyed an equivalent amount of information. For instance, "S. V. is a fisherman. He uses the shell game to trick unsuspecting tourists" would convey a history of cheating. "T. N. is a chauffeur. He works for a leading politician of the opposition party" would convey behavior that is irrelevant to the cheating-trustworthiness dimension. "T. L. is a construction worker. He volunteered to build a home for refugee children" would convey trustworthy behavior. (The stimulus descriptions are available from the authors upon request.)

An item-based analysis showed that the number of words (see Table 1) did not differ among conditions; that is, between cheating and trustworthiness, $t(22)=-0.22, p=.83, \eta_{\mathrm{p}}^{2}<.01$; between cheating and irrelevant behavior, $t(22)=-0.27, p=.79, \eta_{\mathrm{p}}^{2}<.01$; and between trustworthiness and irrelevant behavior, $t(22)<0.01$, $p>.99, \eta_{\mathrm{p}}^{2}<.01$. As in Experiment 1, the descriptions were decomposed into propositions by two raters with expertise in propositional scoring to assess the amount of information provided by these descriptions. The number of propositions did not differ among conditions; that is, between cheating and trustworthiness, $t(22)=0.60, p=.56, \eta_{\mathrm{p}}^{2}=.02$; between cheating and irrelevant behavior, $t(22)<0.01, p>.99, \eta_{\mathrm{p}}^{2}<.01$; and between trustworthiness and irrelevant behavior, $t(22)=-0.60, p=.56, \eta_{\mathrm{p}}^{2}=.02$ (see Table 1).

Information about the valence and the concreteness of the descriptions was obtained in a single independent norming study $(N=25$; see Table 1$)$. Valence was assessed on a scale ranging from -3 (negative) to +3 (positive). Cheating was perceived as more negative than the irrelevant behavior, $t(22)=-15.74, p<$ $.01, \eta_{\mathrm{p}}^{2}=.92$, and trustworthiness was perceived as more positive than the irrelevant behavior, $t(22)=15.20, p<.01, \eta_{\mathrm{p}}^{2}=.91$. In terms of absolute valence (i.e., ignoring the minus sign for the descriptions of cheating), descriptions of cheating and trustworthiness were equally valent, $t(22)=0.51, p=.62, \eta_{\mathrm{p}}^{2}=.01$. Concreteness did not differ among conditions; that is, between cheating and trustworthiness, $t(22)=-0.10, p=.92, \eta_{\mathrm{p}}^{2}<.01$; between cheating and irrelevant behavior, $t(22)=0.19, p=.85$, $\eta_{\mathrm{p}}^{2}<.01$; and between trustworthiness and irrelevant behavior, $t(22)=0.27, p=.79, \eta_{\mathrm{p}}^{2}<.01$.

Given a sample size of $N=47$, and $\alpha=.05$, the power to detect a difference between the source memory parameters for faces of 
cheaters and irrelevant faces $\left(d_{\mathrm{C}}\right.$ and $\left.d_{\mathrm{I}}\right)$ with an effect size of $w=$ 0.06 - which is in the order of magnitude of the source memory effect observed by Buchner et al. (2009) —was reasonably large (1 $-\beta=.94)$. The same applies to the general linear model withinsubject comparisons. Given the assumptions spelled out in Experiment 1 , effects of size $f=.23$ could be detected with the probability of $1-\beta=.95$.

\section{Results}

Encoding-phase likability ratings. Encoding-phase likability ratings varied as a function of behavioral history, $F(2,45)=$ 73.05, $p<.01, \eta_{\mathrm{p}}^{2}=.77$. Orthogonal contrasts showed that cheaters were less likable than other characters, $F(1,46)=149.34$, $p<.01, \eta_{\mathrm{p}}^{2}=.77$, and trustworthy characters were more likable than irrelevant characters, $F(1,46)=46.57, p<.01, \eta_{\mathrm{p}}^{2}=.50$. Mean likability ratings were $2.29(S E=0.08)$ for cheaters, 3.46 $(S E=0.08)$ for irrelevant characters, and $4.08(S E=0.11)$ for trustworthy characters.

Old-new recognition. Replicating Experiment 1 and most previous studies (Barclay \& Lalumière, 2006; Bell \& Buchner, 2009, 2010a, 2011; Buchner et al., 2009; Mehl \& Buchner, 2008), old-new recognition did not differ among the different types of faces, $F(2,45)=0.89, p=.42, \eta_{\mathrm{p}}^{2}=.04$. The mean hit rate was $.69(S E=.03)$ for faces of cheaters, $.65(S E=.03)$ for faces of irrelevant characters, and $.67(S E=.03)$ for faces of trustworthy characters. The mean false alarm rate to new items was .03 ( $S E=$ $.01)$.

Global source memory. To analyze source classification performance, we again computed the unbiased hit rate $H_{\mathrm{u}}$ (Wagner, 1993) for selecting the correct behavior category in the source memory task. As in Experiment 1, there was a significant main effect of behavioral history, $F(2,45)=3.42, p=.04, \eta_{\mathrm{p}}^{2}=.13$. Source classification performance was better for faces of cheaters than for faces of irrelevant characters, $F(1,46)=6.49, p=.01, \eta_{\mathrm{p}}^{2}=.12$, but did not differ significantly between faces of trustworthy and faces of irrelevant characters, $F(1,46)=3.70, p=.06, \eta_{\mathrm{p}}^{2}=.07$.

Next, we tested whether the global source memory advantage for faces of cheaters would be confirmed when Bayen et al.'s (1996) source-monitoring model (see Figure 1) was used. The base model characterized by the assumption that the parameters representing the probability of detecting old faces equal the parameter representing the probability of detecting new faces $\left(D_{\mathrm{C}}=D_{\mathrm{I}}=\right.$ $\left.D_{\mathrm{T}}=D_{\mathrm{N}}\right)$ again fit the data, $G^{2}(3)=5.68, p=.12$. Panel B of Figure 2 shows that the source memory parameters followed the same pattern as in Experiment 1. Equating source memory parameters for faces of cheaters and faces of irrelevant characters $\left(d_{\mathrm{C}}=\right.$ $d_{\mathrm{I}}$ ) resulted in a significant decrease in model fit, $\Delta G^{2}(1)=11.07$, $p<.01$. Thus, there was a source memory advantage for faces of cheaters over faces of irrelevant persons. Source memory did not differ between faces of trustworthy persons and faces of irrelevant persons, $\Delta G^{2}(1)=1.01, p=.32$. These results replicate those obtained in Experiment 1.

Partial and specific source memory. We again assessed whether participants recalled the gist of the behavior description accompanying the face at encoding using a lenient gist-based scoring criterion. Memory for the specific behavior accompanying a face at encoding was again low and did not differ significantly among conditions, $F(2,45)=2.49, p=.09, \eta_{\mathrm{p}}^{2}=.10$. Descrip- tively, the tendency was again toward better memory for the descriptions of cheating faces (see Table 3).

The model of partial and specific source memory illustrated in Figure 3 was again used to assess separately the availability of partial source memory for the behavior category and specific source memory for the behavior description accompanying the face at encoding. The base model implying that all parameters representing the probability of detecting old faces as old can be equated with the probability of detecting new faces as new $\left(D_{\mathrm{C}}=\right.$ $D_{\mathrm{I}}=D_{\mathrm{T}}=D_{\mathrm{N}}$ ) again fit the data, $G^{2}(12)=13.41, p=.34$. Partial source memory for the behavior category was somewhat worse than in Experiment 1 (see Figure 4). Nevertheless, the parameters for partial source memory followed the same pattern as in Experiment 1. Partial source memory for faces of cheaters and for faces of trustworthy characters was above zero $\left(\Delta G^{2}=27.25\right.$, bootstrap $p<.01$, and $\Delta G^{2}=3.22$, bootstrap $p=.04$, respectively) Consistent with the hypothesis that partial source memory reflects categorical emotional tagging, participants had no partial source memory for (nonemotional) irrelevant characters $\left(\Delta G^{2}=0.10\right.$, bootstrap $p=.37$ ). Partial source memory was again clearly better for faces of cheaters than for faces of irrelevant characters, $\Delta G^{2}(1)=7.75, p<.01$, and did not differ between trustworthy and irrelevant faces, $\Delta G^{2}(1)=0.89, p=.34$.

Next, we analyzed specific source memory (see Figure 4). As in Experiment 1, specific source memory was above zero in all three conditions (i.e., the restrictions that $S_{\mathrm{C}}=0, S_{\mathrm{I}}=0$, and $S_{\mathrm{T}}=0$ were incompatible with the data, $\Delta G^{2}=124.40$, bootstrap $p<$ $.01 ; \Delta G^{2}=70.73$, bootstrap $p<.01 ;$ and $\Delta G^{2}=56.10$, bootstrap $p<.01$, respectively). As with partial source memory, the absolute level of specific source memory was descriptively somewhat worse than in Experiment 1. However, given that a different sample of participants was examined at a different time (i.e., participants were not assigned randomly to one of the two experiments), interpreting absolute parameter values seems to be problematic. The relative sizes of the parameters are more important. Specific source memory was better for cheaters than for irrelevant persons, $\Delta G^{2}(1)=5.98, p=.01$, but did not differ between trustworthy and irrelevant persons, $\Delta G^{2}(1)=0.15, p=.69$. Thus, consistent with our expectations, the contribution of specific source memory to the global source memory advantage for faces of cheaters seems to be somewhat larger in Experiment 2 than in Experiment 1 . Nevertheless, the probability of remembering the specific content of the behavior description was again quite low. Thus, the source memory advantage for faces of cheaters is largely caused by unspecific memory for the behavior category, although a small part of the source memory advantage may be caused by memory of more specific information.

Propositional recall. Again, propositional recall was low (see Table 3 ) and did not differ among conditions, $F(2,45)=2.14$, $p=.13, \eta_{\mathrm{p}}^{2}=.09$. This finding corroborates the finding of Experiment 1 that the source memory advantage for faces of cheaters was not accompanied by a more detailed recollection of the behavior descriptions.

\section{Discussion}

In Experiment 2, the global source memory advantage for faces of cheaters was replicated with stimulus material that was even more carefully controlled than that used previously. Again, we 
were interested in separating source memory for the emotional context information into specific source memory for individual behavior descriptions and partial source memory for the emotional categories implied by these descriptions. The first thing that should be noticed is the striking similarity of the results of the two experiments at a descriptive level, confirming the robustness of these findings. Experiment 2 is inconsistent with the hypothesis that partial source memory for the emotional contexts is restricted to situations in which the amount of available information places exceptionally high constraints on memory. Although we drastically reduced the length and complexity of the behavior descriptions, the pattern of results was similar to that obtained in Experiment 1 . The parameters for partial source memory followed the same pattern as the global source memory parameters that were obtained using Bayen et al.'s (1996) source-monitoring model. Partial source memory for faces of cheaters was significantly enhanced. These findings confirm the conclusion reached for Experiment 1 that the source memory advantage for faces of cheaters is largely due to participants' remembering a categorical cheater tag without recollecting the specific type of cheating behavior associated with the face. Although there was a small advantage for memory of the specific aspects of the cheating descriptions in the multinomial analysis, specific source memory was again generally poor. An analysis of propositional recall confirmed that almost no details of the behavior descriptions were remembered.

\section{General Discussion}

The present results can be summarized as follows: (1) As in previous studies (Bell \& Buchner, 2010a, 2010b; Buchner et al., 2009), emotional context had no effect on old-new face recognition; (2) the global source memory advantage for faces associated with negative context information observed in previous experiments (Bell \& Buchner, 2010a, 2010b; Buchner et al., 2009) was replicated; (3) a cued recall test showed that participants nevertheless had almost no memory for the specific details of the context information, showing that they remembered a condensed summary of the behavior descriptions in the form of a categorical emotional tag; and (4) in Experiment 2, the amount of context information was drastically reduced to examine whether emotional tagging is a strategy that is used only when the available information drastically exceeds cognitive resources. Although there was a small advantage for specific source memory of cheating contexts in the multinomial analysis of Experiment 2, the general pattern of results was similar to that obtained in Experiment 1. The source memory advantage was largely due to unspecific memory for categorical emotional information.

These results contribute to our understanding of the source memory enhancement for emotional context information. There are several routes through which emotion may facilitate source memory judgments. In some situations, negative emotion may indeed increase the vividness and detailedness of memory. This was observed in previous studies in which enhanced source memory for emotional material was associated with enhanced recollection of this material (Kensinger \& Corkin, 2003). A possible explanation for this type of source memory enhancement is that negative, especially threatening, information captures attentional resources (Wentura, Rothermund, \& Bak, 2000), which facilitates encoding of the emotional details of the learning episode. This may explain why enhanced source memory for emo- tional stimuli is often accompanied by the feeling of recollection that is expressed in "remember" judgments in the remember-know procedure (Kensinger \& Corkin, 2003). A similar mechanism may account for the small cheater advantage for specific source information that was observed in the multinomial analysis of the results of Experiment 2 and the descriptive trend toward better specific source memory for negative information in Experiment 1 . However, given that source memory for the specific behavior descriptions was generally poor in both experiments, and given that the cheater advantage for specific source information was not statistically significant in Experiment 1, it may be appropriate to conclude that the source memory advantage for negative context information in the present paradigm cannot solely be attributed to better memory for the specific behavior descriptions. The analysis of propositional recall confirms this conclusion by showing that memory for the details of the behavior descriptions was generally poor and did not differ among conditions.

These findings show that a source memory advantage for emotional information is not always accompanied by an enhanced recollection of the specific details of the context information. When examining the effects of emotion on source memory, it may be useful to distinguish between memory for the nonemotional context in which an emotional item was presented and memory for the emotional context in which a nonemotional item was presented. For the former, it has been shown that source memory is enhanced only when the emotional item and the emotionally neutral context are perceptually or conceptually integrated to form a cohesive experience (Kensinger, 2007, 2009; Mather, 2007). For instance, the emotional content of a word may enhance source memory for font color because the context attribute is perceptually integrated into the emotionally arousing stimulus (Mather, 2007). This type of source memory may coincide with a subjectively more vivid recollective experience (Kensinger \& Corkin, 2003). Source memory for the emotional context in which an emotionally neutral stimulus was presented may also depend on whether the stimulus and its context are perceptually or conceptually integrated. In a recent experiment (Bell \& Buchner, 2011), we found enhanced source memory for faces of cheaters but virtually no source memory for faces of victims of cheating. This suggests that source memory for faces is enhanced only to the degree to which the emotional context determines the emotional evaluation of the face. The present results suggest that this type of source memory enhancement is not (always) due to memory for the specific details of the context information. Instead, the source memory advantage for faces of cheaters seems to be due to people's remembering the emotional connotations of the context information without recollecting more specific details about the context information. A recent experiment using the same paradigm (Bell et al., 2010) showed that people are able to discriminate between two types of negative source information (e.g., cheating and disgusting behavior). Thus, people do not only remember the emotional valence of the stimulus face on an evaluative good-bad dimension but rather remember a condensed summary of the behavior description in the form of a categorical emotional tag.

This type of emotional source memory may differ qualitatively from nonemotional source memory that is commonly assessed in source monitoring tasks. Consistent with this interpretation, Kensinger and Schacter (2006) found that source memory for the emotional context in which nonemotional items were presented depended 
on hippocampal activity but not on emotion-processing brain regions. In contrast, Smith and colleagues (Smith, Henson, Dolan, \& Rugg, 2004; Smith, Henson, Rugg, \& Dolan, 2005) found that the source memory advantage for neutral objects superimposed on emotional scenes was accompanied by activity in emotion-processing brain circuits at encoding and retrieval. As in the present experiments, correct source judgments could be based on an awareness of the prior emotional association without recollection of the specific context details. A study examining the brain activation correlates of source memory for faces of cheaters (Singer, Kiebel, Winston, Dolan, \& Frith, 2004) yielded consistent results.

The observation that source memory for faces of cheaters is due to unspecific emotional categorizations fits with the general view that long-term memory typically contains only the gist of the learning episode and lacks specific detail. The memory advantage for faces of cheaters has previously been attributed to a highly specialized cheater identification module closely tied to the faceprocessing system (e.g., Mealey et al., 1996). However, evidence showing that the source memory advantage generalizes to other types of stimuli (Bell \& Buchner, 2009) and other types of threatening information (Bell \& Buchner, 2010b) converges in the conclusion that the source memory advantage is caused by more general effects of emotion on memory. Although the results so far do not support the assumption of a highly specialized cheater identification module, it may nevertheless be illuminating to discuss the present results from a functional perspective (e.g., Nairne, 2010; Nairne \& Pandeirada, 2010) within the context of theories of reciprocal altruism (Axelrod \& Hamilton, 1981; Trivers, 1971). Given that prosocial and antisocial behavior is often considered to be situation-specific (e.g., Zimbardo, 2004) and cross-situational stability of cheating is questionable (e.g., Leming, 1978), a functional perspective would have led to the prediction that the specific type of cheating should have been remembered in more detail, which was not confirmed by the results reported here.

However, a huge advantage of an unspecific memory mechanism is that the behaviorally relevant essence of the learning episode - that a particular person is not to be trusted - is remembered with minimal cognitive resources. Partial source memory is known to be less dependent on the availability of constrained cognitive resources than is specific source memory. For instance, Dodson et al. (1998) showed that dividing attention during retrieval affected specific but not partial source memory. Generalizing these findings to the present results, one may assume that partial source memory for the association between a person and the associated emotion is less fragile and less dependent on the availability of attentional resources at retrieval than is the nonemotional source memory that is commonly assessed. Consistent with this assumption, Johnson, Kim, and Risse (1985) found that Korsakoff syndrome patients preferred photographs of men that were previously characterized by favorable information over photographs of men characterized by unfavorable information although they had virtually no memory for the descriptions that were paired with the photographs. Parallel to these findings, Rahhal, May, and Hasher (2002) reported that older adults had difficulties remembering whether the presentation of a face was accompanied by a female or a male voice. However, when participants were informed that the voice was diagnostic of the depicted person's character, older adults remembered the emotional context as accurately as did younger adults. Similar dissociations have been shown between other types of source memory for socioemotional and perceptual context details (May,
Rahhal, Berry, \& Leighton, 2005; Rahhal et al., 2002). Confirming the robustness of emotional source memory, we observed emotional source memory in an incidental learning situation in which recollection of the specific context details was generally low, and Buchner et al. (2009) showed surprisingly good source memory for faces of cheaters after a 1-week retention interval.

Reducing the load on memory resources is particularly useful when available information overloads cognitive resources and may be quite useful in social exchange. In most societies, people live in large social networks of multiple individuals interacting repeatedly with each other. Based on such considerations, it has recently been argued (Stevens, Volstorf, Schooler, \& Rieskamp, 2010; Volstorf, Rieskamp, \& Stevens, 2011) that social exchange strategies relying on memory for specific encounters are error-prone because they place too high demands on memory. Social exchange strategies implying the classification of potential interaction partners into categories based on summaries of different behavior episodes seems adaptive because they take the limits of memory resources into account. Similarly, Klein et al. (2002) argued that integrating detailed information from different episodes into a summary judgment would take too much time to be of use in most social interactions, which typically require fast decisions. Therefore, it may be beneficial to store general summary judgments about the persons' characters with clear behavior implications. Speculatively, partial source memory for faces of cheaters (without memory for the specific cheating behavior) may induce a tendency of overgeneralized avoidance of cheaters regardless of the specific type of social exchange situation. Obviously, such a tendency would minimize the risk of being cheated twice by the same person in different contexts. As a side effect, overgeneralized avoidance of cheaters might function as a means to punish cheaters by social exclusion and might thereby contribute to the establishment of social norms within a group.

An interesting question is why source memory was significantly enhanced only for faces of cheaters but source memory for trustworthy persons was only descriptively enhanced. This pattern replicates the results obtained in previous experiments (Bell \& Buchner, 2010a; Buchner et al., 2009). The same negativepositive asymmetry was also observed when source memory for other types of negative and positive contexts (i.e., descriptions of disgusting and pleasant behaviors) was obtained (Bell \& Buchner, 2010b). One possible interpretation is that this negativity advantage may be due to a general negativity or threat bias in human information processing (Baumeister, Bratslavsky, Finkenauer, \& Vohs, 2001). This bias seems to be adaptive because of a general asymmetry in the long-term consequences of negative and positive events (Baumeister et al., 2001; Peeters \& Czapinski, 1990; Pratto \& John, 1991). Several studies have shown that negatively valenced-and especially threatening-information recruits more attention (Buchner, Rothermund, Wentura, \& Mehl, 2004; Pratto \& John, 1991) and is better remembered (Kensinger, Garoff-Eaton, \& Schacter, 2007; Ochsner, 2000) than positive information. The negative-positive asymmetry in partial source memory obtained in the present experiments may be related to a negativity bias in the weighting of negative and positive information in social information processing. Negative behaviors that are threatening to observers seem to be generally perceived as being more diagnostic of internal behavior traits than are positive behaviors (Skowronski, 2002; Skowronski \& Carlston, 1987, 1989). Thus, people may be 
biased toward categorizing other people in a negative way. Given these general negative-positive asymmetries in emotional information processing, it is not surprising that categorical emotional tagging is more readily elicited by negative information.

In summary, the present study examined the specificity of source memory for emotional context information. Two types of memory were assessed: (1) partial source memory for categorical emotional information and (2) specific source memory for the details of the context information. Although there was a small advantage of negative context information for specific source memory in Experiment 2, the results are generally consistent with the assumption that the source memory advantage for faces of cheaters is due to unspecific memory for categorical emotional information rather than to recollection of the specific details of the context information. At a more general level, the present results confirm the conclusion derived from previous studies (Dodson et al., 1998; Hicks et al., 2002; Klauer \& Wegener, 1998; Meiser \& Sattler, 2007) that source memory is not always accompanied by a more vivid recollection but may also reflect vague memory for partial or incomplete source information.

\section{References}

Anderson, L., \& Shimamura, A. P. (2005). Influences of emotion on context memory while viewing film clips. American Journal of Psychology, 118, 323-337.

Axelrod, R., \& Hamilton, W. D. (1981, March 27). The evolution of cooperation. Science, 211, 1390-1396. doi:10.1126/science.7466396

Barclay, P., \& Lalumière, M. L. (2006). Do people differentially remember cheaters? Human Nature, 17, 98-113. doi:10.1007/s12110-006-1022-y

Baumeister, R. F., Bratslavsky, E., Finkenauer, C., \& Vohs, K. D. (2001). Bad is stronger than good. Review of General Psychology, 5, 323-370. doi:10.1037/1089-2680.5.4.323

Bayen, U. J., \& Kuhlmann, B. G. (2011). Influences of source-item contingency and schematic knowledge on source monitoring: Tests of the probability-matching account. Journal of Memory and Language, 64, 1-17. doi:10.1016/j.jml.2010.09.001

Bayen, U. J., Murnane, K., \& Erdfelder, E. (1996). Source discrimination, item detection, and multinomial models of source monitoring. Journal of Experimental Psychology: Learning, Memory, and Cognition, 22, 197215. doi:10.1037/0278-7393.22.1.197

Bayen, U. J., Nakamura, G. V., Dupuis, S. E., \& Yang, C. L. (2000). The use of schematic knowledge about sources in source monitoring. Memory \& Cognition, 28, 480-500. doi:10.3758/BF03198562

Bell, R., \& Buchner, A. (2009). Enhanced source memory for names of cheaters. Evolutionary Psychology, 7, 317-330.

Bell, R., \& Buchner, A. (2010a). Justice sensitivity and source memory for cheaters. Journal of Research in Personality, 44, 677-683. doi:10.1016/ j.jrp.2010.08.011

Bell, R., \& Buchner, A. (2010b). Valence modulates source memory for faces. Memory \& Cognition, 38, 29-41. doi:10.3758/MC.38.1.29

Bell, R., \& Buchner, A. (2011). Source memory for faces is determined by their emotional evaluation. Emotion, 11, 249-261. doi:10.1037/a0022597

Bell, R., Buchner, A., \& Mund, I. (2008). Age-related differences in irrelevant-speech effects. Psychology and Aging, 23, 377-391. doi: 10.1037/0882-7974.23.2.377

Bell, R., Giang, T., \& Buchner, A. (2010). Partial and specific source memory for faces associated to other- and self-relevant negative contexts. Manuscript submitted for publication.

Biemann, C., Bordag, S., Heyer, G., Quasthoff, U., \& Wolff, C. (2004). Language-independent methods for compiling monolingual lexical data. In A. Gelbukh (Ed.), Computational linguistics and intelligent text processing (pp. 217-228). Berlin, Germany: Springer. doi:10.1007/9783-540-24630-5_27

Bröder, A., \& Meiser, T. (2007). Measuring source memory. Zeitschrift fur Psychologie, 215, 52-60.

Buchner, A., Bell, R., Mehl, B., \& Musch, J. (2009). No enhanced recognition memory, but better source memory for faces of cheaters. Evolution and Human Behavior, 30, 212-224. doi:10.1016/j.evolhumbehav .2009.01.004

Buchner, A., Rothermund, K., Wentura, D., \& Mehl, B. (2004). Valence of distractor words increases the effects of irrelevant speech on serial recall. Memory \& Cognition, 32, 722-731. doi:10.3758/BF03195862

Conway, M. A., \& Dewhurst, S. A. (1995). Remembering, familiarity, and source monitoring. Quarterly Journal of Experimental Psychology A: Human Experimental Psychology, 48, 125-140.

Cook, G. I., Hicks, J. L., \& Marsh, R. L. (2007). Source monitoring is not always enhanced for valenced material. Memory \& Cognition, 35, 222230. doi:10.3758/BF03193443

Cosmides, L., \& Tooby, J. (1992). Cognitive adaptations for social exchange. In J. H. Barkow, L. Cosmides, \& J. Tooby (Eds.), The adapted mind: Evolutionary psychology and the generation of culture (pp. 163228). New York, NY: Oxford University Press.

D'Argembeau, A., \& Van der Linden, M. (2004). Influence of affective meaning on memory for contextual information. Emotion, 4, 173-188. doi:10.1037/1528-3542.4.2.173

Dodson, C. S., Holland, P. W., \& Shimamura, A. P. (1998). On the recollection of specific- and partial-source information. Journal of Experimental Psychology: Learning, Memory, and Cognition, 24, 11211136. doi:10.1037/0278-7393.24.5.1121

Doerksen, S., \& Shimamura, A. P. (2001). Source memory enhancement for emotional words. Emotion, 1, 5-11. doi:10.1037/1528-3542.1.1.5

Ehrenberg, K., \& Klauer, K. C. (2005). Flexible use of source information: Processing components of the inconsistency effect in person memory. Journal of Experimental Social Psychology, 41, 369-387. doi:10.1016/ j.jesp.2004.08.001

Erdfelder, E., Auer, T.-S., Hilbig, B. E., Aßfalg, A., Moshagen, M., \& Nadarevic, L. (2009). Multinomial processing tree models: A review of the literature. Zeitschrift für Psychologie, 217, 108-124.

Faul, F., Erdfelder, E., Lang, A. G., \& Buchner, A. (2007). G*Power 3: A flexible statistical power analysis program for the social, behavioral, and biomedical sciences. Behavior Research Methods, 39, 175-191. doi: 10.3758/BF03193146

Fiske, S. T., \& Neuberg, S. L. (1990). A continuum of impression formation, from category-based to individuating processes: Influences of information and motivation on attention and interpretation. In M. Zanna (Ed.), Advances in experimental social psychology (pp. 1-74). San Diego, CA: Academic Press.

Hicks, J. L., Marsh, R. L., \& Ritschel, L. (2002). The role of recollection and partial information in source monitoring. Journal of Experimental Psychology: Learning, Memory, and Cognition, 28, 503-508. doi: 10.1037/0278-7393.28.3.503

Johnson, M. K., Kim, J. K., \& Risse, G. (1985). Do alcoholic Korsakoff's syndrome patients acquire affective reactions? Journal of Experimental Psychology: Learning, Memory, and Cognition, 11, 22-36. doi:10.1037/ 0278-7393.11.1.22

Kensinger, E. A. (2007). Negative emotion enhances memory accuracy: Behavioral and neuroimaging evidence. Current Directions in Psychological Science, 16, 213-218. doi:10.1111/j.1467-8721.2007.00506.x

Kensinger, E. A. (2009). Remembering the details: Effects of emotion. Emotion Review, 1, 99-113.

Kensinger, E. A., \& Corkin, S. (2003). Memory enhancement for emotional words: Are emotional words more vividly remembered than neutral words? Memory \& Cognition, 31, 1169-1180. doi:10.3758/BF03195800

Kensinger, E. A., Garoff-Eaton, R. J., \& Schacter, D. L. (2006). Memory for specific visual details can be enhanced by negative arousing content. 
Journal of Memory and Language, 54, 99-112. doi:10.1016/ j.jml.2005.05.005

Kensinger, E. A., Garoff-Eaton, R. J., \& Schacter, D. L. (2007). Effects of emotion on memory specificity in young and older adults. Journal of Gerontology B: Psychological Sciences, 62, 208-215.

Kensinger, E. A., \& Schacter, D. L. (2006). Amygdala activity is associated with the successful encoding of item, but not source, information for positive and negative stimuli. Journal of Neuroscience, 26, 25642570. doi:10.1523/JNEUROSCI.5241-05.2006

Kintsch, W., \& van Dijk, T. A. (1978). Toward a model of text comprehension and production. Psychological Review, 85, 363-394. doi: 10.1037/0033-295X.85.5.363

Klauer, K. C., \& Ehrenberg, K. (2005). Social categorization and fit detection under cognitive load: Efficient or effortful? European Journal of Social Psychology, 35, 493-516. doi:10.1002/ejsp.266

Klauer, K. C., \& Kellen, D. (2010). Toward a complete decision model of item and source recognition: A discrete-state approach. Psychonomic Bulletin \& Review, 17, 465-478. doi:10.3758/PBR.17.4.465

Klauer, K. C., \& Oberauer, K. (1995). Testing the mental model theory of propositional reasoning. Quarterly Journal of Experimental Psychology A: Human Experimental Psychology, 48, 671-687.

Klauer, K. C., \& Wegener, I. (1998). Unraveling social categorization in the "who said what?" paradigm. Journal of Personality and Social Psychology, 75, 1155-1178. doi:10.1037/0022-3514.75.5.1155

Klauer, K. C., Wegener, I., \& Ehrenberg, K. (2002). Perceiving minority members as individuals: The effects of relative group size in social categorization. European Journal of Social Psychology, 32, 223-245. doi:10.1002/ejsp.68

Klein, S. B., Cosmides, L., Tooby, J., \& Chance, S. (2002). Decisions and the evolution of memory: Multiple systems, multiple functions. Psychological Review, 109, 306-329. doi:10.1037/0033-295X.109.2.306

Landis, J. R., \& Koch, G. G. (1977). The measurement of observer agreement for categorical data. Biometrics, 33, 159-174. doi:10.2307/2529310

Leming, J. S. (1978). Cheating behavior, situational influence, and moral development. Journal of Educational Research, 71, 214-217.

Macrae, C. N., \& Bodenhausen, G. V. (2000). Social cognition: Thinking categorically about others. Annual Review of Psychology, 51, 93-120. doi:10.1146/annurev.psych.51.1.93

Mather, M. (2007). Emotional arousal and memory binding: An objectbased framework. Perspectives on Psychological Science, 2, 33-52. doi:10.1111/j.1745-6916.2007.00028.x

Mather, M., Mitchell, K. J., Raye, C. L., Novak, D. L., Greene, E. J., \& Johnson, M. K. (2006). Emotional arousal can impair feature binding in working memory. Journal of Cognitive Neuroscience, 18, 614-625. doi:10.1162/jocn.2006.18.4.614

May, C. P., Rahhal, T., Berry, E. M., \& Leighton, E. A. (2005). Aging, source memory, and emotion. Psychology and Aging, 20, 571-578. doi:10.1037/0882-7974.20.4.571

Mealey, L., Daood, C., \& Krage, M. (1996). Enhanced memory for faces of cheaters. Ethology and Sociobiology, 17, 119-128. doi:10.1016/ 0162-3095(95)00131-X

Mehl, B., \& Buchner, A. (2008). No enhanced memory for faces of cheaters. Evolution and Human Behavior, 29, 35-41. doi:10.1016/ j.evolhumbehav.2007.08.001

Meiser, T., \& Bröder, A. (2002). Memory for multidimensional source information. Journal of Experimental Psychology: Learning, Memory, and Cognition, 28, 116-137. doi:10.1037/0278-7393.28.1.116

Meiser, T., \& Sattler, C. (2007). Boundaries of the relation between conscious recollection and source memory for perceptual details. Consciousness and Cognition, 16, 189-210. doi:10.1016/j.concog.2006.04.003

Meiser, T., Sattler, C., \& Weisser, K. (2008). Binding of multidimensional context information as a distinctive characteristic of remember judgments. Journal of Experimental Psychology: Learning, Memory, and Cognition, 34, 32-49. doi:10.1037/0278-7393.34.1.32
Moshagen, M. (2010). multiTree: A computer program for the analysis of multinomial processing tree models. Behavior Research Methods, 42, 42-54. doi:10.3758/BRM.42.1.42

Mund, I., Bell, R., \& Buchner, A. (in press). Aging and interference in story recall. Experimental Aging Research.

Murnane, K., \& Bayen, U. J. (1996). An evaluation of empirical measures of source identification. Memory \& Cognition, 24, 417-428. doi: 10.3758/BF03200931

Nairne, J. S. (2010). Adaptive memory: Evolutionary constraints on remembering. In B. H. Ross (Ed.), The psychology of learning and motivation: Advances in research and theory (Vol. 53, pp. 1-32). San Diego, CA: Elsevier Academic Press.

Nairne, J. S., \& Pandeirada, J. N. S. (2010). Adaptive memory: Nature's criterion and the functionalist agenda. American Journal of Psychology, 123, 381-390.

Ochsner, K. N. (2000). Are affective events richly recollected or simply familiar? The experience and process of recognizing feelings past. Journal of Experimental Psychology: General, 129, 242-261. doi: 10.1037/0096-3445.129.2.242

Peeters, G., \& Czapinski, J. (1990). Positive-negative asymmetry in evaluations: The distinction between affective and informational negativity effects. In W. Stroebe \& M. Hewstone (Eds.), European review of social psychology (Vol. 1, pp. 33-60). Chichester, England: Wiley.

Perfect, T. J., Mayes, A. R., Downes, J. J., \& Van Eijk, R. (1996). Does context discriminate recollection from familiarity in recognition memory? Quarterly Journal of Experimental Psychology A: Human Experimental Psychology, 49, 797-813.

Pratto, F., \& John, O. P. (1991). Automatic vigilance: The attentiongrabbing power of negative social information. Journal of Personality and Social Psychology, 61, 380-391. doi:10.1037/0022-3514.61.3.380

Rahhal, T. A., May, C. P., \& Hasher, L. (2002). Truth and character: Sources that older adults can remember. Psychological Science, 13, 101-105. doi:10.1111/1467-9280.00419

Riefer, D. M., \& Batchelder, W. H. (1988). Multinomial modeling and the measurement of cognitive processes. Psychological Review, 95, 318 339. doi:10.1037/0033-295X.95.3.318

Riefer, D. M., Chien, Y., \& Reimer, J. F. (2007). Positive and negative generation effects in source monitoring. Quarterly Journal of Experimental Psychology, 60, 1389-1405. doi:10.1080/17470210601025646

Schmitt, M., Gollwitzer, M., Maes, J., \& Arbach, D. (2005). Justice sensitivity: Assessment and location in the personality space. European Journal of Psychological Assessment, 21, 202-211. doi:10.1027/10155759.21.3.202

Schütz, J., \& Bröder, A. (2011). Signal detection and threshold models of source memory. Experimental Psychology, 58, 293-311. doi:10.1027/ 1618-3169/a000097

Singer, T., Kiebel, S. J., Winston, J. S., Dolan, R. J., \& Frith, C. D. (2004). Brain responses to the acquired moral status of faces. Neuron, 41, 653-662. doi:10.1016/S0896-6273(04)00014-5

Skowronski, J. J. (2002). Honesty and intelligence judgments of individuals and groups: The effects of entity-related behavior diagnosticity and implicit theories. Social Cognition, 20, 136-169. doi:10.1521/ soco.20.2.136.20993

Skowronski, J. J., \& Carlston, D. E. (1987). Social judgment and social memory: The role of cue diagnosticity in negativity, positivity, and extremity biases. Journal of Personality and Social Psychology, 52, 689-699. doi:10.1037/0022-3514.52.4.689

Skowronski, J. J., \& Carlston, D. E. (1989). Negativity and extremity biases in impression formation: A review of explanations. Psychological Bulletin, 105, 131-142. doi:10.1037/0033-2909.105.1.131

Smith, A. P. R., Henson, R. N. A., Dolan, R. J., \& Rugg, M. D. (2004) fMRI correlates of the episodic retrieval of emotional contexts. NeuroImage, 22, 868-878. doi:10.1016/j.neuroimage.2004.01.049

Smith, A. P. R., Henson, R. N. A., Rugg, M. D., \& Dolan, R. J. (2005) 
Modulation of retrieval processing reflects accuracy of emotional source memory. Learning \& Memory, 12, 472-479. doi:10.1101/lm.84305

Snodgrass, J. G., \& Corwin, J. (1988). Pragmatics of measuring recognition memory: Applications to dementia and amnesia. Journal of Experimental Psychology: General, 117, 34-50. doi:10.1037/0096-3445.117.1.34

Spaniol, J., \& Bayen, U. J. (2002). When is schematic knowledge used in source monitoring? Journal of Experimental Psychology: Learning, Memory, and Cognition, 28, 631-651. doi:10.1037/0278-7393.28.4.631

Stevens, J. R., Volstorf, J., Schooler, L. J., \& Rieskamp, J. (2010). Forgetting constrains the emergence of cooperative decision strategies. Frontiers in Psychology, 1, 235.

Suzuki, A., \& Suga, S. (2010). Enhanced memory for the wolf in sheep's clothing: Facial trustworthiness modulates face-trait associative memory. Cognition, 117, 224-229. doi:10.1016/j.cognition.2010.08.004

Trivers, R. L. (1971). The evolution of reciprocal altruism. Quarterly Review of Biology, 46, 35-57. doi:10.1086/406755

Turner, A., \& Greene, E. (1977). The construction and use of a propositional text base (Technical Report No. 63). Boulder, CO: Institute for the Study of Intellectual Behavior, University of Colorado.

Van Knippenberg, A., \& Dijksterhuis, A. (2000). Social categorization and stereotyping: A functional perspective. In W. Stroebe \& M. Hewstone
(Eds.), European review of social psychology (Vol. 11, pp. 105-144). Chichester, England: Wiley.

Vogt, V., \& Bröder, A. (2007). Independent retrieval of source dimensions: An extension of results by Starns and Hicks (2005) and a comment on the ACSIM measure. Journal of Experimental Psychology: Learning, Memory, and Cognition, 33, 443-450. doi:10.1037/0278-7393.33.2 .443

Volstorf, J., Rieskamp, J., \& Stevens, J. R. (2011). The good, the bad, and the rare: Memory for partners in social interactions. PLoS One, 6, e18945. doi:10.1371/journal.pone.0018945

Wagner, H. L. (1993). On measuring performance in category judgment studies on nonverbal behavior. Journal of Nonverbal Behavior, 17, 3-28. doi:10.1007/BF00987006

Wentura, D., Rothermund, K., \& Bak, P. (2000). Automatic vigilance: The attention-grabbing power of approach- and avoidance-related social information. Journal of Personality and Social Psychology, 78, 10241037. doi:10.1037/0022-3514.78.6.1024

Zimbardo, P. G. (2004). A situationist perspective on the psychology of evil: Understanding how good people are transformed into perpetrators. In A. Miller (Ed.), The social psychology of good and evil (pp. 21-50). New York, NY: Guilford Press.

\section{Appendix}

\section{Parameter Estimates for Parameters Representing Old-New Recognition, Source Memory, and Guessing Biases}

Table A1

\begin{tabular}{lcc}
\hline \multicolumn{1}{c}{ Parameter } & Experiment 1 & Experiment 2 \\
\hline Old-new recognition & & \\
$D_{\mathrm{C}}=D_{\mathrm{I}}=D_{\mathrm{T}}=D_{\mathrm{N}}$ & .59 & .64 \\
Specific source memory & & .10 \\
$S_{\mathrm{C}}$ & .13 & .05 \\
$S_{\mathrm{I}}$ & .09 & .06 \\
$S_{\mathrm{T}}$ & .09 & .23 \\
Partial source memory & & .02 \\
$P_{\mathrm{C}}$ & .28 & .11 \\
$P_{\mathrm{I}}$ & .03 & .22 \\
$P_{\mathrm{T}}$ & .14 & .07 \\
Guessing biases & & .20 \\
$G_{\mathrm{C}}$ & .32 & .09 \\
$G_{\mathrm{I}}$ & .11 & .62 \\
$G_{\mathrm{T}}$ & .25 & .40 \\
$b$ & .10 & .36 \\
$a_{\mathrm{CT}}$ & .66 & .55 \\
$g_{\mathrm{CT}}$ & .54 & .42 \\
$a_{\mathrm{C}}$ & .45 & .52 \\
$g_{\mathrm{C}}$ & & .25 \\
\hline
\end{tabular}

Note. $\quad D=$ probability of correctly identifying a face as old (with subscripts $\mathrm{C}, \mathrm{I}$, and T representing cheater, irrelevant, and trustworthy, respectively) or new (with subscript $\mathrm{N}$ representing new); $S=$ probability of recalling the specific behavior description accompanying a face at encoding; $P=$ probability of partial source memory for the behavior category in absence of memory for the specific behavior description; $G=$ probability of guessing a random behavioral description when the specific behavior the face was associated with is not known; $b=$ probability of guessing that a face was "old"; $a_{\mathrm{CT}}=$ probability of guessing that a recognized face was encountered in a socially relevant context (cheating or trustworthiness); $g_{\mathrm{CT}}=$ probability of guessing that a nonrecognized face was encountered in a socially relevant context; $a_{\mathrm{C}}=$ probability of guessing that a recognized face belonged to a cheater; $g_{\mathrm{C}}=$ probability of guessing that a nonrecognized face belonged to a cheater.

Received May 3, 2011 